\title{
ANISOTROPIC MIXED FINITE ELEMENTS FOR ELASTICITY
}

\author{
A. PECHSTEIN ${ }^{1}$ AND J. SCHÖBERL ${ }^{2}$
}

\begin{abstract}
In the present paper, we present a family of mixed finite elements which are suitable for the discretization of slim domains. The displacement space is chosen as Nédélec's space of tangential continuous elements, while the stress is approximated by normal-normal continuous symmetric tensor-valued finite elements. We show stability of the system on a slim domain discretized by a tensor product mesh, where the constant of stability does not depend on the aspect ratio of the discretization. We give interpolation operators for the finite element spaces, and thereby obtain optimal order a-priori error estimates for the approximate solution. All estimates are independent of the aspect ratio of the finite elements.
\end{abstract}

\section{INTRODUCTION}

In this paper, we are concerned with finite element simulations for anisotropic structures such as thin plates or shells. For regular domains, there are many methods available to discretize and solve the problem of linearized elasticity, for example Galerkin formulations using continuous finite elements [11], or more involved mixed formulations $[2,4,3]$. However, when the aspect ratio of the computational domain deteriorates, severe problems arise when using standard methods for elasticity. The stability of these methods depends on Korn's inequality $[26,17]$, where the constant is proportional to the aspect ratio of the domain. Thus, the method suffers from locking, which results in poor approximation properties.

To overcome these problems, beam, plate or shell models were introduced. Examples are the Kirchhoff [36] and Reissner-Mindlin plate model $[27,21]$. A widely used technique for shells is the Koiter model [19]. A main drawback of conventional plate or shell elements is the fact that they include not only displacement- and stress-related degrees of freedom, but also rotational quantities. Thus, coupling to standard finite elements in well-shaped parts of the domain becomes difficult,

Key words and phrases. Mixed Finite Elements, Elasticity, Shear Locking, Shells. 
and is often done by brute-force constraints which destroy convergence properties of the respective methods. Also iterative solution techniques have to be handled with care in the presence of rotational degrees of freedom.

A basic concept in order to derive suitable models for thin structures is the idea of hierarchical modeling. There, the behavior of stresses and displacements is taken polynomial in the thickness variable. One obtains a hierarchy of discretizations by increasing the order of the polynomial ansatz, which is related to the $p$-version of a three-dimensional finite element method. Such models were first introduced in variational form by [37]. An overview over different discretization techniques for anisotropic structures within a unified framework is provided in [20]. Analysis for the hierarchical approach can be found in $[1,10,15]$, aposteriori error estimates in [5, 29, 32].

In [33, 31], we first introduced a mixed, Hellinger-Reissner type method, where the stresses are considered as separate unknowns. We searched for the displacement in $H$ (curl), using tangential-continuous Nédélec finite elements. For the stresses, we proposed the space $H$ (div div), discretized by symmetric tensor-valued elements with continuous normal component of the normal stress vector. The degrees of freedom for these elements are then tangential component of the displacement and normal component of the normal stress vector, which shall be abbreviated by normal-normal stress. In the present paper, we apply this approach to a prismatic, tensor-product mesh. We see that these elements do not suffer from shear locking: In the discrete setting, we can use a "broken norm" of piecewise strains for the displacement. Employing appropriate transformations of the finite element shape functions from the reference element to an element in the mesh, we overcome the difficulties arising from Korn's inequality. We provide anisotropic interpolation operators for the stress and displacement spaces with respect to the broken norms. There we make use of the tensor product structure and Clément-type interpolators $[14,30]$ which satisfy a commuting diagram property.

1.1. Basic notations. For some Hilbert space $X$, let $(., .)_{X}$ be the inner product, and $\|.\|_{X}$ the induced norm. By angles $\langle., .\rangle_{X}$, we denote the duality product between the dual space $X^{*}$ and $X$. For some sufficiently regular domain $\Omega \subset \mathbb{R}^{3}$, let $L^{2}(\Omega)$ be the Lebesgue space. As a short hand for the $L^{2}$ norm $\|\cdot\|_{L^{2}(\Omega)}$, we also use $\|\cdot\|_{\Omega}$. By $H^{k}(\Omega)$, integer $k>0$ we denote the standard Sobolev space, with semi-norm 
$|\cdot|_{H^{k}(\Omega)}$ and norm $\|\cdot\|_{H^{k}(\Omega)}$ defined via

$$
|v|_{H^{k}(\Omega)}:=\left\|\nabla^{k} v\right\|_{L^{2}(\Omega)}, \quad\|v\|_{H^{k}(\Omega)}^{2}:=\|v\|_{L^{2}(\Omega)}^{2}+|v|_{H^{k}(\Omega)}^{2} .
$$

Let $H_{0}^{k}(\Omega)$ be the subspace satisfying homogenous boundary conditions for the function and its first $k-1$ derivatives. Then $H^{-k}(\Omega):=H_{0}^{k}(\Omega)^{*}$ is its dual space. We use the spaces

$$
\begin{aligned}
H(\operatorname{curl}, \Omega) & :=\left\{v \in\left[L^{2}(\Omega)\right]^{3}: \operatorname{curl} v \in\left[L^{2}(\Omega)\right]^{3}\right\}, \\
H(\operatorname{div}, \Omega) & :=\left\{q \in\left[L^{2}(\Omega)\right]^{3}: \operatorname{div} q \in L^{2}(\Omega)\right\} .
\end{aligned}
$$

There the operators curl, div are defined via

$$
\operatorname{curl} v:=\nabla \times v, \quad \operatorname{div} v=\nabla \cdot v
$$

and always understood in weak sense. For a tensor-valued function $\sigma$, the divergence operator is defined by row-wise application. We call the tensor symmetric, if it is equal to its transpose, $\sigma=\sigma^{T}$. We abbreviate the space of symmetric tensor-valued $L^{2}$-fields on $\Omega$ by $L_{\text {sym }}^{2}(\Omega)$. Similarly, $H_{\text {sym }}(\operatorname{div}, \Omega)=[H(\operatorname{div}, \Omega)]^{3} \cap L_{\text {sym }}^{2}(\Omega)$ contains the symmetric tensor fields with divergence in $\left[L^{2}(\Omega)\right]^{3}$ in the weak sense. Finally, let $\mathcal{C}(\Omega)$ and $\mathcal{C}(\bar{\Omega})$ be the space of continuous functions on the open respectively closed domains $\Omega$ and $\bar{\Omega}$. On a union of domains $\mathcal{O}=\bigcup_{i \in \mathcal{I}} \Omega_{i}$, all above spaces are defined piecewise without any continuity assumptions. If clear from the context, the domain $\Omega$ can also be omitted.

Let now $\Gamma \subset \bar{\Omega}$ be an oriented, bounded, sufficiently smooth twodimensional manifold, and $\mathcal{G}=\bigcup_{i \in \mathcal{I}} \Gamma_{i}$ a union of such manifolds. We again need the Lebesgue spaces $L^{2}(\Gamma), L^{2}(\mathcal{G})$. Let $n_{\Gamma}$ denote the unique unit normal of $\Gamma$, and let $n_{\Omega}$ be the outward unit normal on the boundary $\partial \Omega$. For better readability, we will drop the subscripts $\Omega, \Gamma$ whenever possible. On such a manifold with normal $n$, we define the normal and tangential component $v_{n}, v_{\tau}$ of a vector valued function $v$ by

$$
v=v_{n} n+v_{\tau}, \quad v_{n}:=v \cdot n .
$$

A tensor field $\sigma$ has a normal vector $\sigma_{n}:=\sigma n$. The normal and tangential parts $\sigma_{n n}, \sigma_{n \tau}$ of this vector are defined by

$$
\sigma_{n}=\sigma_{n n} n+\sigma_{n \tau}, \quad \sigma_{n n}:=n^{T} \sigma n .
$$

Figure 1 displays these normal and tangential components. For a twodimensional vector $v=\left(v_{x_{1}}, v_{x_{2}}\right)^{T} \in \mathbb{R}^{2}$, its normal is defined by $v^{\perp}:=$ $\left(-v_{x_{2}}, v_{x_{1}}\right)^{T}$.

Let now $\Gamma$ be the common interface of two domains $\Omega_{1}, \Omega_{2}$, without loss of generality we assume its normal $n$ to be directed outward of $\Omega_{1}, n=n_{\Gamma}=n_{\Omega_{1}}$. For a sketch of the setting, see Figure 2. On $\Gamma$, the brackets $\llbracket \cdot \rrbracket_{\Gamma}$ shall denote the jump of a discontinuous function 

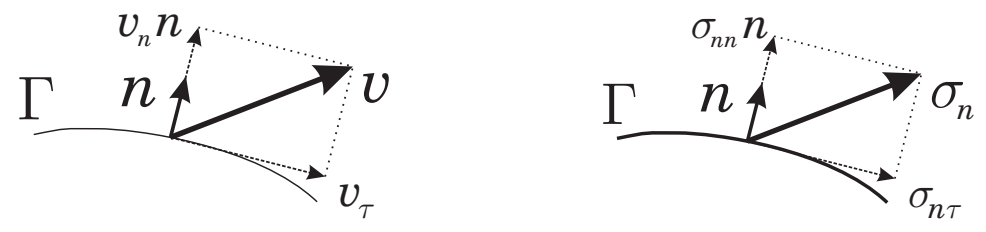

FiguRE 1. Normal and tangential components of a vector $v$ and normal tensor component $\sigma_{n}$ with respect to the manifold $\Gamma$.

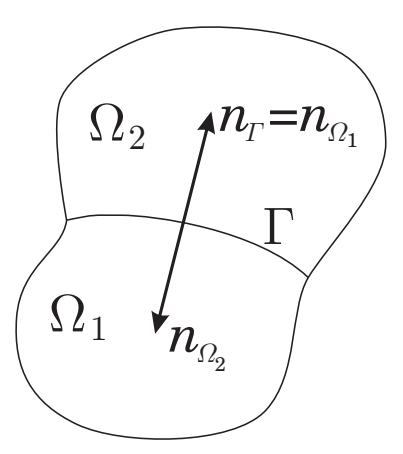

Figure 2. Two domains $\Omega_{1}, \Omega_{2}$ with common interface $\Gamma$. The normal $n_{\Gamma}$ is directed outward of $\Omega_{1}$.

across this interface. We denote the jump in normal direction by $\llbracket \cdot \rrbracket_{n}$, precisely

$$
\llbracket v \rrbracket_{\Gamma}=\left.v\right|_{\Omega_{1}}-\left.v\right|_{\Omega_{2}}, \quad \llbracket v \rrbracket_{n}=\left.v_{n_{\Omega_{1}}}\right|_{\Omega_{1}}+\left.v_{n_{\Omega_{2}}}\right|_{\Omega_{2}}=\llbracket v_{n} \rrbracket_{\Gamma} .
$$

Again, $\Gamma$ can be omitted as an index.

In this work, we consider a flat, three-dimensional domain $\Omega=\Omega_{\boldsymbol{x}} \times$ $\Omega_{z}$. There the cross section $\Omega_{\boldsymbol{x}} \subset \mathbb{R}^{2}$ is assumed to be a connected Lipschitz domain with polygonal boundary. For $\Omega_{z}=\left(0, d_{z}\right)$, we call $d_{z}$ the thickness of $\Omega$, which is usually considerably smaller than $d_{\boldsymbol{x}}:=$ $\operatorname{diam}\left(\Omega_{x}\right)$. To emphasize the different qualities of the in-plane and transversal direction, we use coordinates $(\boldsymbol{x}, z)=\left(x_{1}, x_{2}, z\right)$. For a vector-valued function $u$, we refer to its components by $\left(u_{\boldsymbol{x}}, u_{z}\right)=$ $\left(u_{x_{1}}, u_{x_{2}}, u_{z}\right)$. A symmetric tensor $\sigma$ can be divided into the diagonal sub-blocks $\sigma_{\boldsymbol{x}}, \sigma_{z}$ and the off-diagonal block $\sigma_{\boldsymbol{x} z}$. We use $|\cdot|$ to denote the absolute value of a scalar, as well as the Euclidean norm of a vector and the Frobenius norm of a tensor.

Throughout this paper, by $a \preceq b$ we mean that there exists a (generic) constant $C$, independent of the aspect ratio of the domain $\Omega$ or underlying anisotropic mesh sizes, such that $a \leq C b$. Similarly, $a \succeq b$ stands for $a \geq C b$, and $a \simeq b$ abbreviates $a \preceq b$ and $b \preceq a$. 
1.2. Linear elasticity. The equations of linearized elasticity are given by Hooke's law (1) and the equilibrium equation (2)

$$
\begin{aligned}
A \sigma & =\varepsilon(u) & & \text { in } \Omega, \\
\operatorname{div} \sigma & =-f & & \text { in } \Omega .
\end{aligned}
$$

Here the vector field $u$ is the unknown displacement, $\varepsilon(u):=\frac{1}{2}(\nabla u+$ $\left.(\nabla u)^{T}\right)$ is the strain, $\sigma$ represents the symmetric stress tensor, and $A$ is the compliance tensor. The boundary $\partial \Omega$ consists of a non-trivial part $\Gamma_{D} \subseteq \partial \Omega$, where displacement boundary conditions are posed, and the remaining part $\Gamma_{T}=\partial \Omega \backslash \Gamma_{D}$, where traction boundary conditions are prescribed. We assume that the displacement $u$ is given on $\Gamma_{D}$, and surface tractions $\sigma_{n}$ are prescribed on $\Gamma_{T}$. For simplicity, let all data be homogenous,

$$
u=0 \text { on } \Gamma_{D}, \quad \sigma_{n}=0 \text { on } \Gamma_{T} .
$$

Otherwise, all data enter naturally into the solution spaces and variational formulations considered below.

As the compliance tensor $A$ is invertible, the stresses can be eliminated, which yields a primal formulation for the displacements only. Then one basically searches for $u \in V_{\text {primal }}:=\left\{v \in\left[H^{1}(\Omega)\right]^{3}: v=\right.$ 0 on $\left.\Gamma_{D}\right\}$, such that

$$
\int_{\Omega}\left(A^{-1} \varepsilon(u)\right): \varepsilon(v) d x=\int_{\Omega} f \cdot v d x \quad \forall v \in V_{\text {primal }} .
$$

One may then use standard nodal finite elements to approximate the displacement $u$. For more detail, see e.g. [11]. The method suffers from shear locking, as the aspect ratio of the domain and the underlying finite element discretization deteriorates.

In [28], mixed formulations for elasticity were introduced. The HellingerReissner formulation of elasticity is to find $u \in V_{\text {mixed }}, \sigma \in \Sigma_{\text {mixed }}$ such that

$$
\begin{aligned}
\int_{\Omega}(A \sigma): \tau d x+\int_{\Omega} \operatorname{div} \tau \cdot u d x & =0 & \forall \tau \in \Sigma_{\text {mixed }} \\
\int_{\Omega} \operatorname{div} \sigma \cdot v d x & =-\int_{\Omega} f \cdot v d x & \forall v \in V_{\text {mixed }} .
\end{aligned}
$$

There, the solution spaces are

$$
\begin{aligned}
V_{\text {mixed }} & :=\left[L^{2}(\Omega)\right]^{3}, \\
\Sigma_{\text {mixed }} & :=\left\{\tau \in H_{\text {sym }}(\operatorname{div}): \tau_{n}=0 \text { on } \Gamma_{T}\right\} .
\end{aligned}
$$

In this formulation, all smoothness assumptions are put onto the stress field, the displacement $u \in\left[L^{2}(\Omega)\right]^{3}$ is left totally discontinuous. In 
order to have a conforming finite element method, one needs to approximate $\sigma$ by symmetric, normal continuous finite elements. This is possible, but only at high computational cost: in $[2,3,6]$, simplicial finite elements for two- and three-dimensional mixed elasticity were constructed. The local dimensions of the respective lowest-order finite elements for the stress space is 24 in two, and 162 in three space dimensions.

In [33], we derived a finite element method which lies in between these two concepts. There,

$$
V:=\left\{v \in H(\operatorname{curl}): v_{\tau}=0 \text { on } \Gamma_{D}\right\}
$$

is chosen as displacement space. This implies to search for the stresses in the tensor-valued space

$$
\Sigma:=\left\{\tau \in H(\operatorname{div} \operatorname{div}): \tau_{n n}=0 \text { on } \Gamma_{T}\right\},
$$

where $H(\operatorname{div} \operatorname{div}, \Omega):=\left\{\tau \in L_{\text {sym }}^{2}(\Omega): \operatorname{div} \operatorname{div} \tau \in H^{-1}(\Omega)\right\}$. Here, the first divergence operator is understood row-wise, while the second one is the standard operator acting on a vector-valued function. In $[7,8]$, it was shown that functions in $H$ (curl) have a tangential trace, which implies that $V$ is well-defined in equation (7). The tangential trace lies in the space $H_{\perp}^{-1 / 2}\left(\operatorname{curl}_{\partial \Omega}, \partial \Omega\right)$, for details see [8]. In [31], we showed that functions from $H$ (div div) have a normal-normal trace, such that for $\sigma \in H$ (div div), $\sigma_{n n}$ is well defined. The proper trace space is $H_{n}^{-1 / 2}(\partial \Omega)$, for an exact definition of this space see [31, Theorem 3.34]. Physically, this normal-normal trace can be understood as the normal component of the surface stress vector.

One finally obtains a variational problem of the standard form

$$
\begin{aligned}
a(\sigma, \tau)+b(\tau, u) & =0 & & \forall \tau \in \Sigma, \\
b(\sigma, v) & & -\langle f, v\rangle & \forall v \in V .
\end{aligned}
$$

There, the bilinear forms are defined via

$$
\begin{aligned}
a(\sigma, \tau) & =\int_{\Omega}(A \sigma): \tau d x, \\
b(\tau, v) & =\langle\operatorname{div} \tau, v\rangle_{H(\mathrm{curl})} .
\end{aligned}
$$

One can then show (see [31]), that the infinite dimensional variational problem (8) is well posed, and there holds the stability estimate

$$
\|\sigma\|_{H(\operatorname{div} \text { div })} \leq C(\Omega)\|f\|_{H(\text { curl })^{*}} .
$$

Let now $\mathcal{T}$ be a triangulation of $\Omega$, and let $\mathcal{E}, \mathcal{F}$ denote the sets of element edges/interfaces, respectively. In two space dimensions, these sets coincide. A piecewise smooth function lies in $H$ (curl), if and only if its tangential component is continuous across interfaces and on edges. 
Therefore, Nédélec elements [24, 25] are the appropriate choice for the discretization. In [31] we showed that it is then necessary to have the stress field normal-normal continuous. We showed, that for $\tau \in$ $H$ (div div), $v \in H$ (curl) piecewise smooth on $\mathcal{T}$, the duality product $b(\tau, v)=\langle\operatorname{div} \tau, v\rangle_{H(\text { curl })}$ can be evaluated by

$$
\begin{aligned}
b(\tau, v) & =\sum_{T \in \mathcal{T}_{h}}\left\{\int_{T} \operatorname{div} \tau \cdot v d x-\int_{\partial T} \tau_{n \tau} \cdot v_{\tau} d s\right\} \\
& =\sum_{T \in \mathcal{T}_{h}}\left\{-\int_{T} \tau: \varepsilon(v) d x+\int_{\partial T} \tau_{n n} v_{n} d s\right\} .
\end{aligned}
$$

Indeed, this characterization holds, as long as the integrals can be understood in the sense of duality products. The equivalence of the integral representations (9), (10) can be shown by integration by parts.

In [31], we provided finite element spaces $\Sigma_{k}, V_{k}$ of arbitrary order $k$ on a simplicial mesh. For these spaces, we saw that the Galerkin approximation of (8) yields a stable system. Moreover, we obtained an optimal order of convergence as the mesh size tended towards zero. In the present paper, we are concerned with finding a similar method for a prismatic, tensor product mesh. We lay special emphasis on the fact that all estimates and constants are independent of the aspect ratio of the finite elements.

This paper is organized as follows: In Section 2, we provide finite element spaces of arbitrary order on a prismatic mesh, which are wellsuited to approximate equation (8). Section 3 is devoted to the stability analysis of the discrete problem, where special care is taken to avoid any dependence on the aspect ratio of the finite elements. Interpolation operators, which lead to a-priori error estimates with respect to the anisotropic mesh-sizes, are presented in Section 4. Finally, Section 5 contains numerical results.

\section{Discretization}

In this section, we find a discretization for the infinite dimensional problem (8). We first specify a tensor product mesh, by choosing shape regular triangulations with respect to the $\boldsymbol{x}$ and $z$ directions separately. On this mesh, we construct a finite element space, exploiting the tensor product structure of the volume discretization.

2.1. Anisotropic triangulation. Let $\mathcal{T}^{x}:=\left\{T^{x}\right\}$ be a shape-regular triangulation of the cross section $\Omega_{\boldsymbol{x}}$, and let $h_{\boldsymbol{x}}$ denote the corresponding mesh size. Moreover, let $\mathcal{T}^{z}:=\left\{T^{z}\right\}$ be a subdivision of $\Omega_{z}$ into segments of maximum length $h_{z}$. For both triangulations, we can define 
a set of element interfaces, which we denote by $\mathcal{F}^{x}$ and $\mathcal{F}^{z}$ respectively. There $\mathcal{F}^{\boldsymbol{x}}:=\left\{F^{\boldsymbol{x}}\right\}$ corresponds to all triangle edges in the plane. In vertical direction, $\mathcal{F}^{z}:=\left\{F^{z}\right\}$ is the set of points defining the subdivision. From this, we derive the tensor product mesh

$$
\mathcal{T}:=\left\{T=T^{x} \times T^{z}: T^{x} \in \mathcal{T}^{x}, T^{z} \in \mathcal{T}^{z}\right\},
$$

which consists of prismatic elements of diameter $h_{\boldsymbol{x}}$ and height $h_{z}$. The set of element interfaces or facets can be divided into an in-plane and a vertical part, addressed by $\mathcal{F}_{\|}, \mathcal{F}_{\perp}$, respectively

$$
\begin{aligned}
\mathcal{F} & :=\mathcal{F}_{\|} \cup \mathcal{F}_{\perp} \\
& =\left\{T^{\boldsymbol{x}} \times F^{z}: T^{\boldsymbol{x}} \in \mathcal{T}^{\boldsymbol{x}}, F^{z} \in \mathcal{F}^{z}\right\} \cup\left\{F^{\boldsymbol{x}} \times T^{z}: F^{\boldsymbol{x}} \in \mathcal{F}^{\boldsymbol{x}}, T^{z} \in \mathcal{T}^{z}\right\} .
\end{aligned}
$$

In-plane facets $F_{\|} \in \mathcal{F}_{\|}$are triangular, whereas vertical facets $F_{\perp} \in \mathcal{F}_{\perp}$ are quadrilaterals.

For an element $T, \Delta_{T}$ denotes the union of all neighboring elements, i.e. elements sharing at least one vertex with $T$. For a facet $F, \Delta_{F}=$ $T_{1} \cup T_{2}$ is the union of the two adjacent elements. In case $F$ is a boundary facet, $\Delta_{F}$ contains only one adjacent element. Define $h_{F}=$ $\left(\left|\Delta_{F}\right|\right) /(2|F|)$ to be the average height of these neighboring elements perpendicular to $F$. For a boundary facet set instead $h_{F}=\left(\left|\Delta_{F}\right|\right) /|F|$. For an in-plane facet $F_{\|}$, this means the height of the adjacent prismatic elements, which implies $h_{F_{\|}} \simeq h_{z}$. For a facet $F_{\perp}$ perpendicular to the cross section, we have $h_{F_{\perp}} \simeq h_{\boldsymbol{x}}$.

2.2. Finite element spaces. In this section, we define anisotropic finite element spaces $V_{k}$ for the displacement space $V$ and $\Sigma_{k}$ for the stress space $\Sigma$ for arbitrary polynomial order $k$.

In order to simplify the notation in the sequel, we agree on a special convention: For an arbitrary vector $\alpha \in \mathbb{R}^{d}$ of dimension $d=1,2$ or 3 , let $T^{\alpha}$ denote a simplex of dimension $d$. Then $P_{\alpha}^{k}\left(T^{\alpha}\right)$ shall be the usual polynomial space in variables $\alpha$ of total degree up to $k$. For $\alpha_{1} \in \mathbb{R}^{d_{1}}, \alpha_{2} \in \mathbb{R}^{d_{2}}$, and two simplices $T^{\alpha_{1}}, T^{\alpha_{2}}$ of dimensions $d_{1}, d_{2}$, let $T=T^{\alpha_{1}} \times T^{\alpha_{2}}$ be the tensor product domain. We define $Q_{\alpha_{1}, \alpha_{2}}^{k_{1}, k_{2}}(T):=P_{\alpha_{1}}^{k_{1}}\left(T^{\alpha_{1}}\right) \otimes P_{\alpha_{2}}^{k_{2}}\left(T^{\alpha_{2}}\right)$ as the space of polynomials of mixed order. On a union $\mathcal{T}$ of such domains, all spaces are defined piecewise, without any continuity conditions. As an example for clarifying the meaning of this notation, we first set $\alpha=z$. Then $d=1$, and $T^{z}$ is the unit segment. $P_{z}^{k}\left(T^{z}\right)$ is the usual polynomial space in $z$ of order up to $k$. For $\alpha=\boldsymbol{x}=\left(x_{1}, x_{2}\right)$ and $T^{\boldsymbol{x}}$ the unit triangle, $P_{\boldsymbol{x}}^{k}\left(T^{\boldsymbol{x}}\right)$ is the polynomial space of total order $k$ on the triangle in variables $x_{1}, x_{2}$. The tensor product space $Q_{\boldsymbol{x}, z}^{k_{1}, k_{2}}\left(T^{\boldsymbol{x}} \times T^{z}\right)$ is the standard polynomial 
space of degree $k_{1}$ with respect to $\boldsymbol{x}$ and degree $k_{2}$ with respect to $z$ on the prism $T^{\boldsymbol{x}} \times T^{z}$.

To derive suitable elements for the volume discretization, we need corresponding elements for the in-plane as well as for the transversal directions. They naturally define tensor-product elements for prisms.

We construct all shape functions on the reference element. We use tangential components of the displacement and normal-normal components of the stress tensor as degrees of freedom. Then, we map the shape functions to an element $T$ in the mesh using transformations which preserve these quantities. For the exact definition of these transformations we refer to Section 2.3. In the global finite element space, they are continuous across interfaces. In [31], it was shown that such a finite element space $\Sigma_{k}$ is appropriate for the discretization of $\Sigma=H$ (div div). Although it is not a strictly conforming space, $\Sigma_{k} \notin \Sigma$, its use was rectified by means of an extensive finite element analysis. It was shown that the pair of spaces allows for optimal order approximation properties when appropriate discrete norms are used.

In the following, we specify the prismatic reference element $\hat{T}$. The hat will denote that the respective quantity is associated to the reference element. For example, we use the local reference coordinates $\hat{\boldsymbol{x}}, \hat{z}$, while for a finite element space $Q, \hat{Q}$ is the space spanned by the shape functions on $\hat{T}$. Let $\hat{T}^{z}$ be the reference segment $(0,1)$ with vertices $\hat{V}_{1}^{z}=0, \hat{V}_{2}^{z}=1$. The reference triangle $\hat{T}^{x}$ has vertices $\hat{V}_{1}^{\boldsymbol{x}}=(1,0), \hat{V}_{2}^{\boldsymbol{x}}=(0,1)$ and $\hat{V}_{3}^{\boldsymbol{x}}=(0,0)$. We introduce barycentric coordinates $\lambda_{m}^{z}, \lambda_{m}^{x}$ with respect to these elements,

$$
\begin{aligned}
& \lambda_{1}^{z}(\hat{z})=1-\hat{z}, \quad \lambda_{2}^{z}(\hat{z})=\hat{z}, \\
& \lambda_{1}^{\boldsymbol{x}}(\hat{\boldsymbol{x}})=\hat{x}_{1}, \quad \lambda_{2}^{\boldsymbol{x}}(\hat{\boldsymbol{x}})=\hat{x}_{2}, \quad \lambda_{3}^{\boldsymbol{x}}(\hat{\boldsymbol{x}})=1-\hat{x}_{1}-\hat{x}_{2} \text {. }
\end{aligned}
$$

Then $\hat{T}=\hat{T}^{x} \times \hat{T}^{z}$ is the reference prism. Figure 3 shows a sketch of the respective elements.

2.2.1. In-plane and transversal finite element spaces. We need the following finite element spaces of order $k$ on the cross section $\Omega_{\boldsymbol{x}}$

$$
\begin{aligned}
\mathcal{L}_{\boldsymbol{x}}^{k} & :=\left\{w \in P_{\boldsymbol{x}}^{k}\left(\mathcal{T}^{\boldsymbol{x}}\right): w \text { continuous }\right\} \\
\mathcal{N}_{\boldsymbol{x}}^{k} & :=\left\{v \in\left[P_{\boldsymbol{x}}^{k}\left(\mathcal{T}^{\boldsymbol{x}}\right)\right]^{2}: v_{\tau} \text { continuous }\right\} \\
\Sigma_{\boldsymbol{x}}^{k} & :=\left\{\tau \in\left[P_{\boldsymbol{x}}^{k}\left(\mathcal{T}^{\boldsymbol{x}}\right)\right]_{s y m}^{2 \times 2}: \tau_{n n} \text { continuous }\right\} \\
\mathcal{P}_{\boldsymbol{x}}^{k} & :=P_{\boldsymbol{x}}^{k}\left(\mathcal{T}^{\boldsymbol{x}}\right)
\end{aligned}
$$



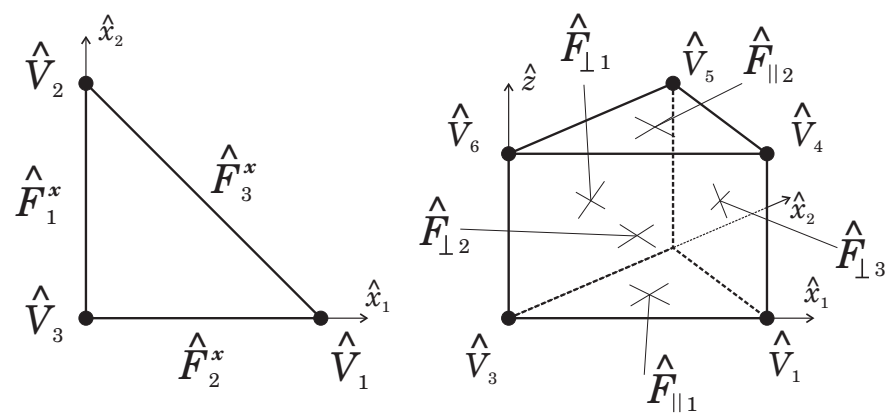

FiguRE 3. Triangular and prismatic reference element

In $z$ direction, we use continuous and non-continuous finite element spaces

$$
\begin{aligned}
\mathcal{L}_{z}^{k} & :=\Sigma_{z}^{k}:=\left\{w \in P_{z}^{k}\left(\mathcal{T}^{z}\right): w \text { cont. }\right\}, \\
\mathcal{P}_{z}^{k} & :=\mathcal{N}_{z}^{k}:=P_{z}^{k}\left(\mathcal{T}^{z}\right) .
\end{aligned}
$$

Most of the spaces defined above are well known: $\mathcal{L}_{x}^{k}, \mathcal{L}_{z}^{k}=\Sigma_{z}^{k}$ are the Lagrange spaces of piecewise polynomial, continuous functions in the plane or on the line, respectively. Also $\mathcal{P}_{\boldsymbol{x}}^{k}, \mathcal{P}_{z}^{k}=\mathcal{N}_{z}^{k}$ are the standard $L^{2}$-conforming spaces of piecewise polynomial functions. Moreover, $\mathcal{N}_{\boldsymbol{x}}^{k}$ is the Nédélec type II space as introduced in [25]. In our analysis and computations, we use the hierarchical basis provided in [35, 38].

We now give an explicit basis for the in-plane space $\hat{\Sigma}_{\boldsymbol{x}}^{k}$ on the reference triangle $\hat{T}^{\boldsymbol{x}}$. Let $\ell_{i}:[-1,1] \rightarrow \mathbb{R}$ be the Legendre polynomial of order $i$. Moreover, we define the scaled Legendre polynomial $\ell_{i}^{S}(\hat{x}, \hat{t}):=\hat{t}^{i} \ell_{i}\left(\frac{\hat{x}}{\hat{t}}\right)$. Using these, we define families of polynomials on the unit segment and triangle, which span different polynomial spaces. There, we use $i, j$ as indices denoting the order of the respective polynomials, whereas $m$ shall correspond to a vertex or edge number. Thus, quantities involving $m$ are always to be seen modulo 3, whereas polynomial orders $i, j$ are assumed to be non-negative.

$$
\begin{aligned}
v_{j}^{z}(z) & :=\ell_{j}\left(1-2 \lambda_{1}^{z}\right), \\
u_{i j}^{\boldsymbol{x}}(\hat{\boldsymbol{x}}) & :=\ell_{i}^{S}\left(\lambda_{1}^{\boldsymbol{x}}-\lambda_{2}^{\boldsymbol{x}}, \lambda_{1}^{\boldsymbol{x}}+\lambda_{2}^{\boldsymbol{x}}\right) \ell_{j}\left(1-2 \lambda_{3}^{\boldsymbol{x}}\right), \\
v_{i}^{\boldsymbol{x}, m}(\boldsymbol{x}) & :=\ell_{i}^{S}\left(\lambda_{m+1}^{\boldsymbol{x}}-\lambda_{m+2}^{\boldsymbol{x}}, \lambda_{m+1}^{\boldsymbol{x}}+\lambda_{m+2}^{\boldsymbol{x}}\right), \quad m=1,2,3 .
\end{aligned}
$$

Then the family $\left\{v_{j}^{z}: 0 \leq j \leq k\right\}$ spans $P_{z}^{k}\left(\hat{T}^{z}\right)$, and $\left\{u_{i j}^{\boldsymbol{x}}, 0 \leq i+j \leq k\right\}$ spans $P_{\boldsymbol{x}}^{k}(\hat{T})$. The scaled Legendre polynomials $\left\{v_{i}^{\boldsymbol{x}, m}: 0 \leq i \leq k\right\}$, restricted to edge $\hat{F}_{m}^{\boldsymbol{x}}$, are a basis for $P_{\boldsymbol{x}}^{k}\left(\hat{F}_{m}^{\boldsymbol{x}}\right)$, which is extended into the interior of the triangle $\hat{T}^{\boldsymbol{x}}$. We now construct symmetric tensor valued basis functions which correspond to the degrees of freedom for 
the normal-normal stress along an element edge. From a direct calculation, one can see that the tensor $\hat{s}_{m}(\hat{\boldsymbol{x}})=\operatorname{sym}\left[\nabla \lambda_{m+1}^{\boldsymbol{x}, \perp} \otimes \nabla \lambda_{m+2}^{\boldsymbol{x}, \perp}\right]$ has a vanishing normal-normal component on two edges, whereas it is constant on the edge $\hat{F}_{m}^{x}$ opposite vertex $m$. Thus, we can define shape functions corresponding to this edge by multiplying $\hat{s}_{m}$ by polynomials spanning $P_{\boldsymbol{x}}^{k}\left(\hat{F}_{m}^{\boldsymbol{x}}\right)$. We define the family of edge basis functions

$$
\hat{\Phi}_{k}^{\hat{F}_{m}^{x}}:=\left\{\hat{s}_{j}^{\hat{F}_{m}^{x}}:=v_{j}^{\boldsymbol{x}, m} \hat{s}_{m}: 0 \leq j \leq k\right\}, \quad m=1,2,3 .
$$

The normal-normal trace of $\hat{\Phi}_{k}^{\hat{F}_{m}^{x}}$ spans $P_{\boldsymbol{x}}^{k}\left(\hat{F}_{m}^{\boldsymbol{x}}\right)$.

Multiplying $\hat{s}_{m}$ with $\hat{b}_{m}^{\boldsymbol{x}}:=\lambda_{m}^{\boldsymbol{x}}$, we obtain an element bubble. This function has vanishing normal-normal trace on all edges of the triangle $\hat{T}$. The family

$$
\hat{\Phi}_{k}^{b, \boldsymbol{x}}:=\left\{\hat{s}_{m i j}^{T^{\boldsymbol{x}}}:=u_{i j}^{\boldsymbol{x}} \hat{b}_{m}^{\boldsymbol{x}} \hat{s}_{m}: 0 \leq i+j \leq k-1, m=1,2,3\right\}
$$

spans the bubble space of order $k$, and is linearly independent [31]. We use the following local space on the reference element

$$
\hat{\Sigma}_{k}^{\boldsymbol{x}}:=\operatorname{span}\left(\bigcup_{m=1}^{3} \hat{\Phi}_{k}^{\hat{F}_{m}^{x}} \cup \hat{\Phi}_{k}^{b, \boldsymbol{x}}\right) .
$$

It is a full polynomial space, $\Sigma_{k}^{\boldsymbol{x}}=P_{\boldsymbol{x}}^{k}\left(\hat{T}^{\boldsymbol{x}}\right)$.

2.2.2. A tensor product finite element space. We propose to use

$$
\begin{aligned}
V_{k}:= & \left\{v \in\left[L^{2}(\Omega)\right]^{3}: v_{\boldsymbol{x}} \in \mathcal{N}_{\boldsymbol{x}}^{k} \otimes \mathcal{L}_{z}^{k+1}, v_{z} \in \mathcal{L}_{\boldsymbol{x}}^{k+1} \otimes \mathcal{N}_{z}^{k}, v_{\tau}=0 \text { on } \Gamma_{D}\right\}, \\
\Sigma_{k}:= & \left\{\tau \in L_{\text {sym }}^{2}(\Omega): \tau_{\boldsymbol{x}} \in \Sigma_{\boldsymbol{x}}^{k} \otimes \mathcal{P}_{z}^{k+1}, \tau_{\boldsymbol{x} z} \in \mathcal{P}_{\boldsymbol{x}}^{k} \otimes \mathcal{P}_{z}^{k}, \tau_{z} \in \mathcal{P}_{\boldsymbol{x}}^{k+1} \otimes \Sigma_{z}^{k+1},\right. \\
& \left.\tau_{n n}=0 \text { on } \Gamma_{T}\right\} .
\end{aligned}
$$

The displacement space defined above coincides with the standard Nédélec space on a tensor product mesh, see e.g. [22, 16]. We concentrate on the construction of basis functions for the stress space on the reference element $\hat{T}$.

Using the constant, in-plane tensors $\hat{s}_{m}$, we can construct tensor fields $\hat{S}_{m}^{\boldsymbol{x}}$ satisfying $\hat{S}_{m, n n}^{\boldsymbol{x}}=0$ on all prism faces but the quadrilateral opposite vertex $m$. By multiplication with $b_{m}^{\boldsymbol{x}}=\lambda_{m}^{\boldsymbol{x}}$, we obtain an element bubble with vanishing normal-normal trace

$$
\hat{S}_{m}^{\boldsymbol{x}}:=\left(\begin{array}{cc}
\hat{s}_{m} & 0 \\
0 & 0
\end{array}\right), \quad \hat{B}_{m}^{\boldsymbol{x}}:=b_{m}^{\boldsymbol{x}} \hat{S}_{m}^{\boldsymbol{x}}, \quad m=1,2,3 .
$$

For the upper and lower triangular face, the unit normal is $n= \pm e_{z}$, and therefore $\hat{\tau}_{n n}=\hat{\tau}_{z}$. The tensor field $\hat{S}^{z}$ defined below has a constant normal-normal component on those two faces, whereas it vanishes on 
the quadrilateral faces. We get a bubble function by multiplication with $b^{z}:=\lambda_{1}^{z} \lambda_{2}^{z}$

$$
\hat{S}^{z}:=\left(\begin{array}{cc}
0 & 0 \\
0 & 1
\end{array}\right), \quad \hat{B}^{z}:=b^{z} \hat{S}^{z}
$$

To span the space of piecewise constant symmetric tensor fields, we need two additional functions $\hat{S}_{m}^{\boldsymbol{x} z}$. These have vanishing normalnormal components on all facets, and are therefore bubble functions (we set $b_{m}^{\boldsymbol{x} z}=1$ )

$$
\hat{S}_{m}^{\boldsymbol{x} z}:=\left(\begin{array}{cc}
0 & e_{x_{m}} \\
e_{x_{m}}^{T} & 0
\end{array}\right), \quad \hat{B}_{m}^{\boldsymbol{x} z}:=b_{m}^{\boldsymbol{x} z} \hat{S}_{m}^{\boldsymbol{x} z}=\hat{S}_{m}^{\boldsymbol{x} z}, \quad m=1,2 .
$$

For convenience, we use $\left\{\hat{B}_{m}, m \in \mathcal{J}_{B}\right\}$ and $\left\{\hat{S}_{m}, m \in \mathcal{J}_{S}\right\}$ for the respective unions of $\hat{B}_{m}^{\boldsymbol{x}}, \hat{B}^{z}, \hat{B}_{m}^{\boldsymbol{x} z}$ and $\hat{S}_{m}^{\boldsymbol{x}}, \hat{S}^{z}, \hat{S}_{m}^{\boldsymbol{x} z}$, whenever we do not care about the special type of the respective tensor field.

We can now introduce finite element spaces $\hat{\Sigma}^{\hat{F}}$ consisting of shape functions associated to each facet $\hat{F}$. This is done multiplying the "facet basis tensors" $\hat{S}_{m}$ by families of polynomials. In case of a triangular facet $\hat{F}_{\|, m}$, this space is

$$
\hat{\Sigma}_{k}^{\hat{F}_{\|, m}}:=\operatorname{span}\left\{u_{i j}^{\boldsymbol{x}}(\hat{\boldsymbol{x}}) \hat{S}^{z} \lambda_{m}^{z}(\hat{z}): 0 \leq i+j \leq k+1\right\} .
$$

For a quadrilateral $\hat{F}_{\perp, m}$ opposite edge $m$, we use

$$
\hat{\Sigma}_{k}^{\hat{F}_{\perp, m}}:=\operatorname{span}\left\{v_{i}^{\boldsymbol{x}, m} v_{j}^{z} \hat{S}_{m}^{\boldsymbol{x}}: 0 \leq i \leq k, 0 \leq l \leq k+1\right\} .
$$

In Section 2.3, we provide a map from the reference element to an element $T$ in the mesh, which preserves normal-normal continuity of the stress tensor. Using this map, it is possible to define the local finite element space $\Sigma_{k}^{F}$ associated to each facet $F \in \mathcal{F}$. Restricted to facet $F$, the normal-normal components of $\Sigma_{k}^{F}$ span a full polynomial space:

$$
\left\{\left.\sigma_{n n}\right|_{F}: \sigma \in \Sigma_{k}^{F}\right\}=\left\{\begin{array}{cl}
P_{\boldsymbol{x}}^{k+1}(F) & \text { if } F \in \mathcal{F}_{\|}, \\
Q_{\boldsymbol{x}, z}^{k, k+1}(F) & \text { if } F \in \mathcal{F}_{\perp} .
\end{array}\right.
$$

The union of these spaces spans the facet space $\Sigma_{k}^{f}:=\bigoplus_{F \in \mathcal{F}} \Sigma_{k}^{F}$.

Finally, we propose a basis for the local bubble space $\hat{\Sigma}_{k}^{b}$. We define

$$
\begin{aligned}
\hat{\Sigma}_{k}^{b}:= & \operatorname{span}\left(\left\{u_{i j}^{\boldsymbol{x}} v_{l}^{z} \hat{B}_{m}^{x}: m=1,2,3,0 \leq i+j \leq k-1,0 \leq l \leq k+1\right\} \cup\right. \\
& \left\{u_{i j}^{x} v_{l}^{z} \hat{B}^{x z}: 0 \leq i+j, l \leq k\right\} \cup \\
& \left.\left\{u_{i j}^{x} v_{l}^{z} \hat{B}_{m}^{z}: m=1,2,0 \leq i+j \leq k+1,0 \leq l \leq k-1\right\}\right) .
\end{aligned}
$$


The two subspaces together build the local finite element space, $\hat{\Sigma}_{k}:=$ $\hat{\Sigma}_{k}^{f}+\hat{\Sigma}_{k}^{b}$. The respective components $\hat{\sigma}_{\boldsymbol{x}}, \hat{\sigma}_{\boldsymbol{x} z}, \hat{\sigma}_{z}$ of $\hat{\sigma} \in \hat{\Sigma}_{k}$ span polynomial spaces,

$$
\hat{\Sigma}_{k}=\left\{\hat{\sigma} \in\left[L^{2}(\Omega)\right]_{\text {sym }}^{3 \times 3}: \hat{\sigma}_{\boldsymbol{x}} \in Q_{\boldsymbol{x}, z}^{k, k+1}, \hat{\sigma}_{\boldsymbol{x} z} \in Q_{\boldsymbol{x}, z}^{k, k}, \hat{\sigma}_{z} \in Q_{\boldsymbol{x}, z}^{k+1, k+1}\right\} .
$$

2.3. Finite element transformations. In the previous section, we provided bases for the finite elements on the reference element $\hat{T}$. We still need to transform these functions to an element $T$ by a conforming transformation, which we describe in detail in the following. First, let $\Phi_{T}: \hat{T} \rightarrow T$ be the mapping from the reference element to an element $T \in \mathcal{T}$. By $F_{T}$, we denote its Jacobian, and by $J_{T}$ the Jacobi determinant. Similarly, for a facet $F$ or an edge $E$, by $J_{F}, J_{E}$ we mean the transformation of measures of the facet transformation $\hat{F} \rightarrow F$ or the edge transformation $\hat{E} \rightarrow E$, respectively. Tangential and normal vectors can be computed from the corresponding reference quantities using the covariant and contravariant or Piola transforms: The unit tangential vector $\tau_{E}$ of an edge $E$ is related to the unit tangent $\hat{\tau}_{\hat{E}}$ of the reference edge $\hat{E}$ via $\tau_{E}=\hat{\tau}_{\hat{E}} F_{T} / J_{E}$. For the unit normal $n_{F}$ of a facet $F$, we have $n_{F}=J_{T} F_{T}^{-T} \hat{n}_{\hat{F}} / J_{F}$.

The transformation $\Phi_{T}$ is linear in $(x, z)$, the Jacobian $F_{T}$ is constant. Moreover, as we use a tensor product mesh, the Jacobian is block diagonal

$$
F_{T}=\left(\begin{array}{cc}
F_{T^{x}} & 0 \\
0 & F_{T^{z}}
\end{array}\right)
$$

The sub-blocks stem from shape-regular triangulations, thus there holds

$$
\left|F_{T^{x}}\right|_{s}^{-1} \simeq\left|F_{T^{x}}^{-1}\right|_{s} \simeq h_{\boldsymbol{x}}^{-1}, \quad\left|F_{T^{z}}\right|_{s}^{-1} \simeq\left|F_{T^{z}}^{-1}\right|_{s} \simeq h_{z}^{-1} .
$$

Here, $|A|_{s}$ denotes the spectral norm of the matrix $A$.

For $(\hat{\boldsymbol{x}}, \hat{z}) \in \hat{T}$ let $(\boldsymbol{x}, z)=\Phi_{T}(\hat{\boldsymbol{x}}, \hat{z})$ be the corresponding point in $T$. Functions $v \in V_{k}$ and $\sigma \in \Sigma_{k}$ are transformed like

$$
\begin{aligned}
v(\boldsymbol{x}, z) & =F_{T}^{-T} \hat{v}(\hat{\boldsymbol{x}}, \hat{z}), \\
\sigma(\boldsymbol{x} z) & =\frac{1}{J_{T}^{2}}\left(F_{T} \hat{\sigma}(\hat{\boldsymbol{x}}, \hat{z}) F_{T}^{T}\right) .
\end{aligned}
$$

For the strain of a vector-valued function $v \in V_{k}$, one can easily see

$$
\varepsilon(v)(\boldsymbol{x} z)=F_{T}^{-1} \hat{\varepsilon}(\hat{v})(\hat{\boldsymbol{x}}, \hat{z}) F_{T}^{-T} .
$$


Note that the following integral values are preserved for $v \in V_{k}, \sigma \in \Sigma_{k}$ and each polynomial $q$,

$$
\begin{aligned}
\int_{E} v_{\tau} q d s & =\int_{\hat{E}} \hat{v} F_{T}^{-1} F_{T} \hat{\tau} q \frac{J_{E}}{J_{E}} d \hat{s}=\int_{\hat{E}} \hat{v}_{\hat{\tau}} q d \hat{s}, \\
\int_{F} \sigma_{n n} J_{F} q d s & =\int_{\hat{F}} \frac{1}{J_{T}^{2}} \hat{n}^{T} \hat{\sigma} \hat{n} \frac{J_{T}^{2}}{J_{F}^{2}} J_{F}^{2} q d \hat{s}=\int_{\hat{F}} \hat{\sigma}_{\hat{n} \hat{n}} q d \hat{s} .
\end{aligned}
$$

We will use these quantities to define degrees of freedom for the respective spaces later on. For the global finite element spaces $V_{k}, \Sigma_{k}$ continuity of these degrees of freedom across inter-element interfaces is required only.

Using the transformations (12), and transforming the shape functions derived before, we obtain finite element spaces $V_{k}, \Sigma_{k}$. For the respective traces on element faces, we observe the following: The tangential component of a displacement $v \in V_{k}$ spans $\left[P_{\boldsymbol{x}}^{k}\right]^{2}$ on a triangular face, and $Q_{\boldsymbol{x}, z}^{k, k+1} \times Q_{\boldsymbol{x}, z}^{k+1, k}$ on a rectangular face. Similarly, the normalnormal component of $\sigma \in \Sigma_{k}$ spans $P_{\boldsymbol{x}}^{k}$ on triangular, as well as $Q_{\boldsymbol{x}, z}^{k, k+1}$ on quadrilateral faces. From the construction of the finite element bases, it is obvious that $\Sigma_{k}$ can be split into a facet and a bubble part, $\Sigma_{k}=\Sigma_{k}^{f}+\Sigma_{k}^{b}$, also globally. There $\Sigma_{k}^{f}$ matches the facet-based space $\hat{\Sigma}_{k}^{f}$ on the reference element, as well as $\Sigma_{k}^{b}$ matches $\hat{\Sigma}_{k}^{b}$.

The discrete problem is to find $v \in V_{k}, \sigma \in \Sigma_{k}$ such that

$$
\begin{array}{rlrl}
a(\sigma, \tau)+b(\tau, u) & =0 & & \forall \tau \in \Sigma_{k}, \\
b(\sigma, v) & =-\langle f, v\rangle \quad \forall v \in V_{k} .
\end{array}
$$

\section{Discrete stability}

In this section, we will see that the discrete mixed problem (13) is well posed, there exists a unique solution, which is bounded by the right hand side. For the analysis of the discrete problem, we do not use the natural norms of $H$ (curl) and $H$ (div div) as in the continuous setting. This is due to the fact that the finite element space $\Sigma_{k}$ is not strictly conforming for $\Sigma=H$ (div div), as shown in [31]. Thus, the natural $H$ (div div) norm does not necessarily exist for the discrete solution. Instead, we use a broken $H^{1}$-type norm for the displacements, as well as the $L^{2}$ norm for the stresses. Precisely, they are given by

$$
\begin{aligned}
\|v\|_{V_{k}}^{2} & :=\sum_{T \in \mathcal{T}}\|\varepsilon(v)\|_{T}^{2}+\sum_{F \in \mathcal{F}} h_{F}^{-1}\left\|\llbracket v \rrbracket_{n}\right\|_{F}^{2}, \\
\|\tau\|_{\Sigma_{k}}^{2} & :=\|\tau\|_{\Omega}^{2} .
\end{aligned}
$$

Note that we do not use piecewise gradients for the displacements, as commonly done for a broken $H^{1}$ norm. Instead, we take piecewise 
strains only. This way we are able to avoid the use of Korn's inequality, which will finally lead to results independent of the aspect ratio of the finite elements. We state that in the scope of this paper, no claims on stability or convergence with respect to the polynomial degree $k$ are made.

The following lemma states a discrete trace inequality for $\Sigma_{k}$ on the element level.

Lemma 1. Let $T \in \mathcal{T}$ be an element with facets $\mathcal{F}_{T}=\{F \in \mathcal{F}: F \subset$ $\partial T\}$.

(1) For $\sigma \in \Sigma_{k}$, there holds the trace inequality

$$
\sum_{F \in \mathcal{F}_{T}} h_{F}\left\|\sigma_{n n}\right\|_{F}^{2} \preceq\|\sigma\|_{T}^{2} .
$$

(2) Let $g$ be in the normal-normal trace space on $\partial T$ of $\Sigma_{k}$, i.e. $g \in P_{\boldsymbol{x}}^{k+1}\left(F_{\|}\right)$for a triangular facet $F_{\|}, g \in Q_{\boldsymbol{x}, z}^{k, k+1}\left(F_{\perp}\right)$ for a quadrilateral facet $F_{\perp}$. Then there exists an extension $\sigma \in \Sigma_{k}$ such that $\sigma_{n n}=g$ on $\partial T$, and

$$
\|\sigma\|_{T}^{2} \preceq \sum_{F \in \mathcal{F}_{T}} h_{F}\|g\|_{F}^{2} .
$$

In both estimates, the constant may depend on the polynomial degree $k$.

Proof.

(1) As described in Section 2.3, we decompose the finite element space $\Sigma_{k}$ into a facet and a bubble part, $\Sigma_{k}=\Sigma_{k}^{f}+\Sigma_{h}^{b}$. For $\sigma \in \Sigma_{k}$, this implies a splitting $\sigma=\sigma^{f}+\sigma^{b}$, where

$$
\sigma^{f}=\sum_{F \in \mathcal{F}} \sigma^{F} \quad \text { and } \quad \sigma^{F} \in \Sigma_{k}^{F} .
$$

Due to the linear independence of the basis functions, on the finite dimensional space there holds

$$
\|\sigma\|_{T} \succeq\left\|\sigma^{f}\right\|_{T}+\left\|\sigma^{b}\right\|_{T} \geq\left\|\sigma^{f}\right\|_{T} .
$$

Let $\sigma^{F}$ be corresponding to face $F$. For an in-plane facet $F_{\|}, \sigma^{F_{\|}}$ is of the special form $\sigma^{F_{\|}}=\left(q / J_{T}^{2}\right) F_{T} \hat{S}^{z} F_{T}^{T}$, where $q$ is a scalarvalued, piecewise polynomial function. Therefore, all entries but $\sigma_{z}^{F_{\|}}$are zero. On the reference element, we have, using the tensor product structure of the element transformation,

$\left|F_{T} \hat{\sigma}^{F_{\|}} F_{T}^{T}\right|^{2}=|q|^{2}\left|F_{T} \hat{S}^{z} F_{T}^{T}\right|^{2} \simeq|q|^{2}\left|F_{\hat{T}^{z}}\right|_{s}^{4} \simeq h_{z}^{4}|q|^{2} \simeq h_{F_{\|}}^{4}\left|\hat{\sigma}^{F_{\|}}\right|^{2}$. 
Recall, that $|\cdot|$ denotes the Frobenius norm, whereas $|\cdot|_{s}$ is the spectral norm of a tensor. On a quadrilateral facet $F_{\perp}$, we obtain similarly for a shape function $\sigma^{F_{\perp}}=\left(q / J_{T}^{2}\right) F_{T} \hat{S}_{i}^{\boldsymbol{x}} F_{T}^{T}$ that

$$
\left|F_{T} \hat{\sigma}^{F_{\perp}} F_{T}^{T}\right|^{2}=|q|^{2}\left|F_{T} \hat{S}_{i}^{\boldsymbol{x}} F_{T}^{T}\right|^{2} \simeq|q|^{2}\left|F_{\hat{T}^{x}}\right|_{s}^{4} \simeq h_{\boldsymbol{x}}^{4}|q|^{2} \simeq h_{F_{\perp}}^{4}\left|\hat{\sigma}^{F_{\perp}}\right|^{2} .
$$

Using these equivalences and the fact that, on the reference element, $\left\|\hat{\sigma}^{F}\right\|_{\hat{T}}$ and $\left\|\hat{\sigma}_{n n}^{F}\right\|_{\hat{F}}$ are equivalent norms due to the finite dimension of the space $\hat{\Sigma}_{k}^{\hat{F}}$, we obtain

$$
\begin{aligned}
\left\|\sigma^{f}\right\|_{T}^{2} & =\int_{T} \sigma^{f}: \sigma^{f} d x=\int_{\hat{T}} \frac{1}{J_{T}^{4}}\left|F_{T} \hat{\sigma}^{f} F_{T}^{T}\right|^{2} J_{T} d \hat{x} \\
& \simeq \sum_{F \in \mathcal{F}_{T}} J_{T}^{-3} h_{F}^{4} \int_{\hat{T}}\left|\hat{\sigma}^{F}\right|^{2} d \hat{x} \\
& \simeq \sum_{F \in \mathcal{F}_{T}} J_{T}^{-3} h_{F}^{4} \int_{\hat{F}}\left|\hat{\sigma}_{\hat{n} \hat{n}}^{F}\right|^{2} d \hat{s} \\
& =\sum_{F \in \mathcal{F}_{T}} J_{T}^{-3} h_{F}^{4} \int_{F} J_{F}^{4}\left|\sigma_{n n}^{F}\right|^{2} \frac{1}{J_{F}} d s .
\end{aligned}
$$

Inserting $h_{F} \simeq J_{T} / J_{F}$ into the estimate above, we get

$$
\left\|\sigma^{f}\right\|_{T}^{2} \simeq \sum_{F \in \mathcal{F}_{T}} h_{F}\left\|\sigma_{n n}^{F}\right\|_{F}^{2}=\sum_{F \in \mathcal{F}_{T}} h_{F}\left\|\sigma_{n n}\right\|_{F}^{2} .
$$

Together with (16), this implies the discrete trace inequality (14).

(2) Let $\sigma^{f}$ be the unique extension of $g$ to the space $\Sigma_{k}^{f}(T)$. Due to the equivalence

$$
\left\|\sigma^{f}\right\|_{T}^{2} \simeq \sum_{F \in \mathcal{F}} h_{F}\left\|\sigma_{n n}^{f}\right\|_{F}^{2}=\sum_{F \in \mathcal{F}} h_{F}\|g\|_{F}^{2}
$$

shown in part (1), we have found the required extension in $\sigma^{f}$.

Lemma 2. The bilinear form $b: \Sigma_{k} \times V_{k} \rightarrow \mathbb{R}$ is inf-sup stable, there exists a positive constant $\widetilde{\beta}>0$ such that

$$
\inf _{v \in V_{k}} \sup _{\sigma \in \Sigma_{k}} \frac{b(\sigma, v)}{\|\sigma\|_{\Sigma_{k}}\|v\|_{V_{k}}} \geq \widetilde{\beta}
$$

The constant $\widetilde{\beta}$ may depend on the polynomial degree $k$, but we explicitely state that $\widetilde{\beta}$ is independent of the anisotropic mesh sizes $h_{\boldsymbol{x}}, h_{z}$ and their ratio. 
Proof. Let $v \in V_{k}$ be given, we want to find $\sigma \in \Sigma_{k}$ such that

$$
b(\sigma, v) \geq \widetilde{\beta}\|\sigma\|_{\Sigma_{k}}\|v\|_{V_{k}}
$$

We construct $\sigma$ as a combination $\alpha \sigma^{f}+\beta \sigma^{b}$, where $\sigma^{f} \in \Sigma_{k}^{f}, \sigma^{b} \in \Sigma_{k}^{b}$, and the constants $\alpha, \beta \in \mathbb{R}$ are to be specified later.

Due to the matching polynomial degrees, we can choose $\sigma^{f}$ such that

$$
\left.\sigma_{n n}^{f}\right|_{F}=-h_{F}^{-1} \llbracket v \rrbracket_{n, F} \quad \forall F \in \mathcal{F} .
$$

From the proof of Lemma 1, we immediately see that there exists a constant $c_{2}>0$ such that

$$
\left\|\sigma^{f}\right\|_{\Sigma_{k}}^{2} \leq c_{2}^{2} \sum_{F \in \mathcal{F}} h_{F}\left\|\sigma_{n n}^{f}\right\|_{F}^{2}=c_{2}^{2} \sum_{F \in \mathcal{F}} h_{F}^{-1}\left\|\llbracket v \rrbracket_{n}\right\|_{F}^{2}
$$

For the definition of $\sigma^{b}$, we need to introduce the block tensor

$$
\tilde{F}_{T}:=\left(\begin{array}{cc}
h_{\boldsymbol{x}} I_{\boldsymbol{x}} & 0 \\
0 & h_{z} I_{z}
\end{array}\right)
$$

where $I_{\boldsymbol{x}}, I_{z}$ denote the identity sub-blocks of the $3 \times 3$ identity matrix $I$. Due to the tensor product structure of the Jacobian $F_{T}$, and the shape-regularity of the in-plane and transversal meshes, we have that $\left|F_{T}\right|_{s} \simeq\left|\tilde{F}_{T}\right|_{s}$ independent of the aspect ratio $h_{\boldsymbol{x}} / h_{z}$. We choose $\sigma^{b}$ such that, on the reference element

$$
\hat{\sigma}^{b}:=J_{T}^{2} \sum_{m \in \mathcal{J}_{B}}\left(\hat{\varepsilon}(\hat{v}): \tilde{F}_{T}^{-T} \hat{S}_{m} \tilde{F}_{T}^{-1}\right) \tilde{F}_{T}^{-T} \hat{B}_{m} \tilde{F}_{T}^{-1}
$$

Obviously, $\hat{\sigma}^{b}$ is a bubble function, i.e. $\hat{\sigma}_{n n}^{b}=0$ on $\partial \hat{T}$. Computing the respective polynomial degrees of its sub-blocks, one verifies $\hat{\sigma}^{b} \in \hat{\Sigma}_{k}$. The tensor field $\sigma^{b}$ is obtained by transformation to element $T$.

We need the following two inequalities

$$
\begin{aligned}
\left\|\sigma^{b}\right\|_{\Sigma_{k}}^{2} & \leq c_{3} \sum_{T \in \mathcal{T}}\|\varepsilon(v)\|_{T}^{2}, \\
\int_{T} \sigma^{b}: \varepsilon(v) d x & \geq c_{1}\|\varepsilon(v)\|_{T}^{2} .
\end{aligned}
$$

The upper bound (19) for $\left\|\sigma^{b}\right\|_{\Sigma_{k}}$ can be shown by a straightforward transformation to the reference element, where one uses the definition of $\sigma^{b}$ and the similarity $\left|F_{T}\right|_{s} \simeq\left|\tilde{F}_{T}\right|_{s}$. We now concentrate on estimate (20). Using that $\left\{\hat{S}_{m}^{\boldsymbol{x}}, \hat{S}^{z}, \hat{S}_{m}^{\boldsymbol{x} z}\right\}$ form a basis for the piecewise constant symmetric tensor fields, that the bubble functions $\left\{\hat{B}_{m}^{\boldsymbol{x}}, \hat{B}^{z}, \hat{B}_{m}^{\boldsymbol{x} z}\right\}$ are 
linearly independent, and the spectral equivalence of $\tilde{F}_{T}$ and $F_{T}$, we obtain

$$
\begin{aligned}
\int_{T} \sigma^{b}: \varepsilon(v) d x & =\int_{\hat{T}} \frac{1}{J_{T}^{2}} \hat{\sigma}^{b}: \hat{\varepsilon}(\hat{v}) J_{T} d \hat{x} \\
& =\int_{\hat{T}} \sum_{m \in \mathcal{J}_{B}}\left(\hat{\varepsilon}(\hat{v}): \tilde{F}_{T}^{-T} \hat{S}_{m} \tilde{F}_{T}^{-1}\right) \tilde{F}_{T}^{-T} \hat{B}_{m} \tilde{F}_{T}^{-1}: \hat{\varepsilon}(\hat{v}) J_{T} d \hat{x} \\
& =\int_{\hat{T}} \sum_{i \in \mathcal{J}_{B}}\left(\tilde{F}_{T}^{-1} \hat{\varepsilon}(\hat{v}) \tilde{F}_{T}^{-T}: \hat{S}_{m}\right)^{2} \hat{b}_{m} J_{T} d x \\
& \simeq \int_{\hat{T}}\left|\tilde{F}_{T}^{-1} \hat{\varepsilon}(\hat{v}) \tilde{F}_{T}^{-T}\right|^{2} J_{T} d \hat{x} \\
& \simeq \int_{\hat{T}}\left|F_{T}^{-1} \hat{\varepsilon}(\hat{v}) F_{T}^{-T}\right|^{2} J_{T} d \hat{x}=\|\varepsilon(v)\|_{T}^{2}
\end{aligned}
$$

We can now show the following lower bound for $b(\sigma, v)$, where we use the estimates from above, as well as Young's inequality in the last line

$$
\begin{aligned}
b(\sigma, v) & =\sum_{T \in \mathcal{T}} \int_{T} \varepsilon(v): \sigma d x-\sum_{F \in \mathcal{F}} \int_{F} \sigma_{n n} \llbracket v \rrbracket_{n} d s \\
& =\sum_{T \in \mathcal{T}} \int_{T} \varepsilon(v):\left(\alpha \sigma^{f}+\beta \sigma^{b}\right) d x-\sum_{F \in \mathcal{F}} \int_{F} \alpha \sigma_{n n}^{f} \llbracket v \rrbracket_{n} d s \\
& \stackrel{(20),(18)}{\geq} \sum_{T \in \mathcal{T}}\left[\beta c_{1}\|\varepsilon(v)\|_{T}^{2}-\alpha\left\|\sigma^{f}\right\|_{T}\|\varepsilon(v)\|_{T}\right]+\sum_{F \in \mathcal{F}} \alpha h_{F}^{-1}\left\|\llbracket v \rrbracket_{n}\right\|_{F}^{2} \\
& \stackrel{(18)}{\geq} \sum_{T \in \mathcal{T}}\left[\beta c_{1}\|\varepsilon(v)\|_{T}^{2}-\sum_{F \subset \partial T} \alpha c_{2} h_{F}^{-1 / 2}\left\|\llbracket v \rrbracket_{n}\right\|_{F}\|\varepsilon(v)\|_{T}\right]+\sum_{F \in \mathcal{F}} \alpha h_{F}^{-1}\left\|\llbracket v \rrbracket_{n}\right\|_{F}^{2} \\
& \geq \sum_{T \in \mathcal{T}}\left(\beta c_{1}-\frac{\alpha c_{2} \gamma^{2}}{2}\right)\|\varepsilon(v)\|_{T}^{2}+\sum_{F \in \mathcal{F}} \alpha h_{F}^{-1}\left(1-\frac{c_{2}}{2 \gamma^{2}}\right)\left\|\llbracket v \rrbracket_{n}\right\|_{F}^{2} .
\end{aligned}
$$

Setting $\gamma^{2}=c_{2}, \alpha=1, \beta=\left(1+c_{2}^{2}\right) /\left(2 c_{1}\right)$, the estimate above together with (18), (19) yields the required result.

We can now prove stability of the discrete problem (13).

Theorem 3. The discrete, mixed system (13) is well-posed. There exists a unique solution $\left(u_{k}, \sigma_{k}\right) \in V_{k} \times \Sigma_{k}$. It satisfies the a-priori error estimate

$$
\left\|u-u_{k}\right\|_{V_{k}}+\left\|\sigma-\sigma_{k}\right\|_{\Sigma_{k}} \leq c\left(\inf _{v_{k} \in V_{k}}\left\|u-v_{k}\right\|_{V_{k}}+\inf _{\tau_{k} \in \Sigma_{k}}\left\|\sigma-\tau_{k}\right\|_{\Sigma_{k}}\right),
$$


where $(u, \sigma)$ denotes the exact solution to the underlying elasticity problem (8). The constant $c>0$ is independent of the anisotropic mesh sizes $h_{\boldsymbol{x}}, h_{z}$ and their ratio $h_{\boldsymbol{x}} / h_{z}$, but may depend on the polynomial degree $k$.

Proof. Following [9, Proposition 2.6, Proposition 2.7], we need boundedness of the bilinear forms $a(\cdot, \cdot), b(\cdot, \cdot)$ with respect to the discrete norms, coercivity of $a(\cdot, \cdot)$ and an inf-sup condition on the finite element spaces for $b(\cdot, \cdot)$. Continuity and coercivity for $a(\cdot, \cdot)$ can easily be shown, as we use the $L^{2}$ norm for $\Sigma_{k}$. Boundedness of $b(\cdot, \cdot)$ follows from Lemma 1, (1). Together with Lemma 2 ensuring inf-sup stability, we obtain the required result.

\section{INTERPOLATION OPERATORS AND ERROR ESTIMATES}

In this section, we propose interpolation operators $I_{k}^{\mathcal{N}}$ for the displacement space $V_{k}$, and $I_{k}^{\Sigma}$ for the stress space $\Sigma_{k}$. Using standard theory for mixed problems, as can be found in [9], interpolation error estimates directly lead to a-priori estimates for the approximation errors $\left\|u-u_{h}\right\|_{V_{k}}$ and $\left\|\sigma-\sigma_{h}\right\|_{\Sigma_{k}}$. For a sufficiently smooth solution $(u, \sigma)$ to problem $(8)$, we achieve the error bound

$$
\begin{aligned}
\left\|\sigma-\sigma_{h}\right\|_{\Sigma_{k}}+\left\|u-u_{h}\right\|_{V_{k}} \preceq & h_{\boldsymbol{x}}^{m}\left(\left\|\nabla_{\boldsymbol{x}}^{m} \sigma\right\|_{L^{2}(\Omega)}+\left\|\nabla_{\boldsymbol{x}}^{m} \varepsilon(u)\right\|_{L^{2}(\Omega)}\right)+ \\
& h_{z}^{m}\left(\left\|\nabla_{z}^{m} \sigma\right\|_{L^{2}(\Omega)}+\left\|\nabla_{z}^{m} \varepsilon(u)\right\|_{L^{2}(\Omega)}\right),
\end{aligned}
$$

where the constant hidden in " $\preceq$ " is independent of $h_{\boldsymbol{x}}, h_{z}$ and the ratio $h_{\boldsymbol{x}} / h_{z}$.

We will see that it is necessary to have the interpolation operator $I_{k}^{\mathcal{N}}$ for the stress space well-defined for $L^{2}$ functions, and bounded with respect to the $L^{2}$ norm. This goal is achieved using quasi-interpolation operators first introduced in [30]. To construct the interpolation operator $I_{k}^{\Sigma}$, we introduce degrees of freedom for $\Sigma_{k}$. These nodal values naturally lead to a nodal interpolation operator. To ensure that all degrees of freedom can be evaluated, we set

$$
\Sigma_{\mathcal{T}}:=\left\{\sigma \in L_{\text {sym }}^{2}(\Omega): \sigma_{n n} \in L^{2}(\mathcal{F}) \text { cont. }\right\} .
$$

4.1. In-plane and transversal interpolation. For $\alpha \in\{\boldsymbol{x}, z\}$, we need interpolation operators

$$
\begin{array}{ll}
I_{\alpha, k}^{\mathcal{L}}: L^{2}\left(\Omega_{\alpha}\right) \rightarrow \mathcal{L}_{\alpha}^{k}, & I_{\alpha, k}^{\mathcal{N}}: L^{2}\left(\Omega_{\alpha}\right) \rightarrow \mathcal{N}_{\alpha}^{k}, \\
I_{\alpha, k}^{\Sigma}: \Sigma_{\mathcal{T}^{\alpha}} \rightarrow \Sigma_{\alpha}^{k}, & I_{\alpha, k}^{\mathcal{P}}: L^{2}\left(\Omega_{\alpha}\right) \rightarrow \mathcal{P}_{\alpha}^{k} .
\end{array}
$$


The last one of these operators, $I_{\alpha, k}^{\mathcal{P}}$, is an element-wise $L^{2}$ projection onto the space of piecewise polynomials. For the stress space, we will introduce the nodal interpolation operator $I_{\alpha, k}^{\Sigma}$ in the end of Section 4.1. The spaces $\mathcal{L}_{\boldsymbol{x}}^{k}, \mathcal{L}_{z}^{k}=\Sigma_{z}^{k}$ are Lagrange spaces of piecewise polynomial, continuous functions. A first idea is to use the nodal Lagrange interpolation operator there (see e.g. [13]). However, this operator is not well defined on $L^{2}\left(\Omega_{\alpha}\right)$, as it needs point evaluation. Similarly, the nodal Nédélec interpolation operator (e.g. provided in [22]) is not well defined on $L^{2}\left(\Omega_{\alpha}\right)$ or even $H\left(\operatorname{curl}, \Omega_{\alpha}\right)$, as it needs to evaluate line integrals along edges of the form $\int_{E} v_{\tau} d s$.

In the $H^{1}$ setting, local averaging operators have been introduced in $[14,34]$ to overcome these difficulties. In [30], quasi-interpolation operators based on the idea of the Clément operator were developed for $H^{1}, H$ (curl), $H(\operatorname{div})$ and $L^{2}$. We will use these for $I_{\alpha, k}^{\mathcal{L}}$ and $I_{\alpha, k}^{\mathcal{N}}$. Note that in [30], they are only provided for the lowest order case. In [31], we showed that all ideas can be transferred directly to operators of arbitrary order. They satisfy standard approximation properties on a shape regular mesh. Let $l, m$ be integers, $l=0,1$ and $l<m \leq k+1$, then we have for sufficiently smooth functions $u, v, q$

$$
\begin{aligned}
\left\|u-I_{\alpha, k}^{\mathcal{L}} u\right\|_{H^{l}\left(T^{\alpha}\right)} & \preceq h_{\alpha}^{m-l}|u|_{H^{m}\left(\Delta_{T^{\alpha}}\right)}, \\
\left\|v-I_{\alpha, k}^{\mathcal{N}} v\right\|_{H^{l}\left(T^{\alpha}\right)} & \preceq h_{\alpha}^{m-l}|v|_{H^{m}\left(\Delta_{T^{\alpha}}\right)}, \\
\left\|q-I_{\alpha, k}^{\mathcal{P}} q\right\|_{H^{l}\left(T^{\alpha}\right)} & \preceq h_{\alpha}^{m-l}|q|_{H^{m}\left(\Delta_{T^{\alpha}}\right)}
\end{aligned}
$$

We note that the interpolation operators above keep the degrees of freedom of the respective spaces for polynomial functions. In the next lemma we show that the strain is approximated by the Nédélec interpolation operator.

Lemma 4. Let $\mathcal{T}^{\alpha}$ be a shape-regular triangulation for $\Omega_{\alpha}$ with $\alpha \in$ $\{\boldsymbol{x}, z\}$, and let us fix an element $T^{\alpha} \in \mathcal{T}^{\alpha}$. For $u \in H^{m+1}\left(\Delta_{T^{\alpha}}\right)$ with $1 \leq m \leq k$, the Nédélec interpolation operator with respect to $\alpha$ satisfies

$$
\left\|\varepsilon_{\alpha}\left(u-I_{\alpha, k}^{\mathcal{N}} u\right)\right\|_{T^{\alpha}} \preceq h_{\alpha}^{m}\left\|\nabla_{\alpha}^{m} \varepsilon_{\alpha}(u)\right\|_{\Delta_{T^{\alpha}}} .
$$

Proof. As $I_{\alpha, k}^{\mathcal{N}}$ preserves polynomials up to order $k$, we have, abbreviating $P^{k}=\left[P^{k}\left(\Delta_{T^{\alpha}}\right)\right]^{\operatorname{dim}(\alpha)}$,

$$
\left\|\varepsilon_{\alpha}\left(u-I_{\alpha, k}^{\mathcal{N}} u\right)\right\|_{T^{\alpha}}=\inf _{q \in P^{k}}\left\|\varepsilon_{\alpha}\left(\left(i d-I_{\alpha, k}^{\mathcal{N}}\right)(u-q)\right)\right\|_{T^{\alpha}} .
$$

The rigid body motions are reproduced by $I_{\alpha, k}^{\mathcal{N}}$. This, and the fact that $P^{k}$ contains the piecewise rigid body motions, ensures that we may employ an inverse inequality for the strain tensor, which relies on 
Korn's inequality on an element of the shape-regular triangulation $\mathcal{T}^{\alpha}$. Together with the $L^{2}$ continuity of $I_{\alpha, k}^{\mathcal{N}}$, we may estimate

$$
\begin{aligned}
\left\|\varepsilon_{\alpha}\left(u-I_{\alpha, k}^{\mathcal{N}} u\right)\right\|_{T^{\alpha}} & \leq \inf _{q \in P^{k}}\left(\left\|\varepsilon_{\alpha}(u-q)\right\|_{T^{\alpha}}+\left\|\varepsilon_{\alpha}\left(I_{\alpha, k}^{\mathcal{N}}(u-q)\right)\right\|_{T^{\alpha}}\right) \\
& \preceq \inf _{q \in P^{k}}\left(h_{\alpha}^{-1}\|u-q\|_{T^{\alpha}}+h_{\alpha}^{-1}\left\|I_{\alpha, k}^{\mathcal{N}}(u-q)\right\|_{T^{\alpha}}\right) \\
& \preceq \inf _{q \in P^{k}} h_{\alpha}^{-1}\|u-q\|_{\Delta_{T^{\alpha}}} .
\end{aligned}
$$

A Bramble-Hilbert argument, see e.g. [18] gives, for $0 \leq m \leq k$

$$
\left\|\varepsilon_{\alpha}\left(u-I_{\alpha, k}^{\mathcal{N}} u\right)\right\|_{T^{\alpha}} \preceq h_{\alpha}^{m+1-1}\left\|\nabla_{\alpha}^{m+1} u\right\|_{\Delta_{T^{\alpha}}} .
$$

In case of $\alpha=\boldsymbol{x}$, one can show $\left\|\nabla_{\boldsymbol{x}}^{m} \varepsilon_{\boldsymbol{x}}(u)\right\|_{\Delta_{T^{x}}} \simeq\left\|\nabla_{\boldsymbol{x}}^{m+1} u\right\|_{\Delta_{T^{\boldsymbol{x}}}}$ for $m \geq 1$ by a direct evaluation of the respective terms. For $\alpha=z$, the strain and gradient operator coincide. Putting these estimates together, we obtain

$$
\left\|\varepsilon_{\alpha}\left(u-I_{\alpha, k}^{\mathcal{N}} u\right)\right\|_{T^{\alpha}} \preceq h_{\alpha}^{m}\left\|\nabla_{\alpha}^{m} \varepsilon_{\alpha}(u)\right\|_{\Delta_{T^{\alpha}}} .
$$

A main achievement of [30] was the statement that the interpolation operators for the Lagrange and Nédélec spaces satisfy a commuting diagram property,

$$
\nabla_{\alpha} I_{\alpha, k+1}^{\mathcal{L}}=I_{\alpha, k}^{\mathcal{N}} \nabla_{\alpha}
$$

This property will be a crucial tool in the analysis of the approximation properties of the tensor product interpolation operator constructed later.

So far, we provided quasi-interpolation operators for all spaces except for the stress space $\Sigma_{\boldsymbol{x}}^{k}$. In the sequel, we propose a nodal interpolation operator for $\Sigma_{\boldsymbol{x}}^{k}$. It is well defined for tensor-valued symmetric $L^{2}$ functions with their normal-normal component continuous across interfaces $F$, and in $L^{2}(F)$. Note that all estimates are done on the shape regular triangulation $\mathcal{T}^{x}$.

To construct a nodal interpolation operator, we need degrees of freedom for $\Sigma_{\boldsymbol{x}}^{k}$. We propose a unisolvent set of degrees of freedom, which are preserved by the $H$ (div div) conforming transformation (12). For $F^{\boldsymbol{x}} \in \mathcal{F}^{\boldsymbol{x}}$ and $T^{\boldsymbol{x}} \in \mathcal{T}^{\boldsymbol{x}}$ they are given by

$$
\begin{aligned}
\Psi_{i}^{F^{\boldsymbol{x}}}\left(\sigma_{\boldsymbol{x}}\right):=\int_{F^{\boldsymbol{x}}} J_{F^{\boldsymbol{x}}} \sigma_{\boldsymbol{x}, n n} \ell_{i} d s_{\boldsymbol{x}}, & 0 \leq i \leq k, \\
\Psi_{m i j}^{T^{\boldsymbol{x}}}\left(\sigma_{\boldsymbol{x}}\right):=\int_{T} J_{T^{\boldsymbol{x}}} \sigma_{\boldsymbol{x}}:\left(F_{T^{x}}^{-T} b_{m}^{\boldsymbol{x}} \hat{s}_{m} F_{T^{\boldsymbol{x}}}^{-1}\right) u_{i j}^{\boldsymbol{x}} d \boldsymbol{x}, & 0 \leq i+j \leq k-1, m=1,2,3 .
\end{aligned}
$$


Note that $b_{m}^{\boldsymbol{x}} \hat{s}_{m}, m=1,2,3$ are the in-plane bubble functions defined in Section 2.2. In [31], it was shown that these functionals are linearly independent.

Thus there exists a unique local polynomial nodal basis $\Phi^{T^{x}}:=$ $\bigcup_{F^{x} \in \mathcal{F}\left(T^{x}\right)}\left\{\phi_{i}^{F^{x}}\right\} \cup\left\{\phi_{i j l}^{T^{x}}\right\}$, which matches these degrees of freedom and spans $P_{\boldsymbol{x}}^{k}\left(T^{\boldsymbol{x}}\right)$ :

$$
\operatorname{span}\left(\Phi^{T^{\boldsymbol{x}}}\right)=P_{\boldsymbol{x}}^{k}\left(T^{\boldsymbol{x}}\right) .
$$

We can now define the nodal interpolator by

$$
\mathcal{I}_{\boldsymbol{x}, k}^{\Sigma}(\sigma):=\sum_{F^{\boldsymbol{x}} \in \mathcal{F}^{x}} \sum_{i=0}^{k} \Psi_{i}^{F^{\boldsymbol{x}}}\left(\sigma_{\boldsymbol{x}}\right) \phi_{i}^{F^{\boldsymbol{x}}}+\sum_{T^{\boldsymbol{x}} \in \mathcal{T}^{x}} \sum_{\substack{0 \leq m \leq 3, 0 \leq i+j \leq k-1}} \Psi_{m i j}^{T^{\boldsymbol{x}}}\left(\sigma_{\boldsymbol{x}}\right) \phi_{m i j}^{T^{\boldsymbol{x}}} .
$$

The interpolation operator preserves polynomials up to order $k$. Note that it naturally commutes with the finite element transformation from Section 2.3. By a Bramble-Hilbert argument on the uniform triangulation, we obtain for sufficiently smooth $\sigma_{\boldsymbol{x}}$ that

$$
\left\|\sigma_{\boldsymbol{x}}-\mathcal{I}_{\boldsymbol{x}, k}^{\Sigma} \sigma_{\boldsymbol{x}}\right\|_{T^{\boldsymbol{x}}} \preceq h_{\boldsymbol{x}}^{m}\left\|\nabla_{\boldsymbol{x}}^{m} \sigma_{\boldsymbol{x}}\right\|_{\Delta_{T^{x}}}, \quad 0 \leq m \leq k+1 .
$$

4.2. Tensor product interpolation operators. We can now define an interpolation operator for the displacement space $V_{k}$ on the tensor product domain,

$$
I_{k}^{\mathcal{N}}:=I_{\boldsymbol{x}, k}^{\mathcal{N}} \otimes I_{z, k+1}^{\mathcal{L}} \times I_{\boldsymbol{x}, k+1}^{\mathcal{L}} \otimes I_{z, k}^{\mathcal{N}} .
$$

The in-plane deformation $u_{\boldsymbol{x}}$ is interpolated by the Nédélec operator in plane, and continuously in the thickness-direction, the transversal displacement $u_{z}$ is interpolated vice versa.

For the stress space, we define, denoting the symmetry of the tensor,

$$
I_{k}^{\Sigma}(\sigma):=\left(\begin{array}{cc}
\left(I_{\boldsymbol{x}, k}^{\Sigma} \otimes I_{z, k+1}^{\mathcal{P}}\right) \sigma_{\boldsymbol{x}} & \left(I_{\boldsymbol{x}, k}^{\mathcal{P}} \otimes I_{z, k}^{\mathcal{P}}\right) \sigma_{\boldsymbol{x} z} \\
\operatorname{sym} & \left(I_{\boldsymbol{x}, k+1}^{\mathcal{P}} \otimes I_{z, k+1}^{\Sigma}\right) \sigma_{z}
\end{array}\right) .
$$

From the definition of the quasi-interpolation operators it is clear that interpolation and differentiation with respect to different directions commute, i.e. for $\alpha, \beta \in\{\boldsymbol{x}, z\}, \alpha \neq \beta$

$$
\nabla_{\alpha} I_{\beta, k}^{\mathcal{L}}=I_{\beta, k}^{\mathcal{L}} \nabla_{\alpha}, \quad \nabla_{\alpha} I_{\beta, k}^{\mathcal{N}}=I_{\beta, k}^{\mathcal{N}} \nabla_{\alpha} .
$$

In the following, we provide two lemmas, that result in an interpolation error estimate for $I_{k}^{\mathcal{N}}$ with respect to the broken norm $\|\cdot\|_{V_{k}}$.

Lemma 5. Let $T \in \mathcal{T}$, and for integer $m \leq k$ let the function $u \in$ $H$ (curl) additionally satisfy $u \in H^{m+1}\left(\Delta_{T}\right)$. Then the product space interpolation operator $I_{k}^{\mathcal{N}}$ defined via (26) satisfies the local anisotropic error estimate for $m \leq k$

$$
\left\|\varepsilon\left(u-I_{k}^{\mathcal{N}} u\right)\right\|_{T} \preceq h_{\boldsymbol{x}}^{m}\left\|\nabla_{\boldsymbol{x}}^{m} \varepsilon(u)\right\|_{\Delta_{T}}+h_{z}^{m}\left\|\nabla_{z}^{m} \varepsilon(u)\right\|_{\Delta_{T}} .
$$


Proof. For $u \in\left[H^{m+1}(T)\right]^{3}$, we bound the three different blocks $\varepsilon_{\boldsymbol{x}}(u), \varepsilon_{z}(u), \varepsilon_{\boldsymbol{x} z}(u)$ of the strain tensor separately. The bounds on the diagonal blocks $\varepsilon_{\boldsymbol{x}}(u), \varepsilon_{z}(u)$ follow straightforwardly and along the same line, we show the estimate for the $\boldsymbol{x}$-block

$$
\begin{aligned}
\frac{1}{2}\left\|\varepsilon_{\boldsymbol{x}}\left(u-I_{\boldsymbol{x}, k}^{\mathcal{N}} I_{z, k+1}^{\mathcal{L}} u\right)\right\|_{T}^{2} & \leq\left\|\left(i d-I_{z, k+1}^{\mathcal{L}}\right) \varepsilon_{\boldsymbol{x}}(u)\right\|_{T}^{2}+\left\|I_{z, p+1}^{\mathcal{L}} \varepsilon_{\boldsymbol{x}}\left(u-I_{\boldsymbol{x}, k}^{\mathcal{N}} u\right)\right\|_{T}^{2} \\
& \preceq h_{z}^{2 m}\left\|\nabla_{z}^{m} \varepsilon_{\boldsymbol{x}}(u)\right\|_{\Delta_{T}}^{2}+\left\|\varepsilon_{\boldsymbol{x}}\left(u-I_{\boldsymbol{x}, k}^{\mathcal{N}} u\right)\right\|_{\Delta_{T}}^{2} \\
& \preceq h_{z}^{2 m}\left\|\nabla_{z}^{m} \varepsilon_{\boldsymbol{x}}(u)\right\|_{\Delta_{T}}^{2}+h_{\boldsymbol{x}}^{2 m}\left\|\nabla_{\boldsymbol{x}}^{m} \varepsilon_{\boldsymbol{x}}(u)\right\|_{\Delta_{T}}^{2} .
\end{aligned}
$$

In the off-diagonal block $\varepsilon_{\boldsymbol{x} z}(u)$, we rely on the commuting diagram property (25) of the quasi-interpolators

$$
\begin{aligned}
\frac{1}{2}\left\|\varepsilon_{\boldsymbol{x} z}\left(u-I_{k}^{\mathcal{N}} u\right)\right\|_{T}^{2} & =\frac{1}{8}\left\|\nabla_{\boldsymbol{x}}\left(u_{z}-I_{z, k}^{\mathcal{N}} I_{\boldsymbol{x}, k+1}^{\mathcal{L}} u_{z}\right)+\nabla_{z}\left(u_{\boldsymbol{x}}-I_{\boldsymbol{x}, k}^{\mathcal{N}} I_{z, k+1}^{\mathcal{L}} u_{\boldsymbol{x}}\right)\right\|_{T}^{2} \\
& =\frac{1}{8}\|\left(i d-I_{\boldsymbol{x}, k}^{\mathcal{N}} I_{z, k}^{\mathcal{N}}\right) \underbrace{\left(\nabla_{\boldsymbol{x}} u_{z}+\nabla_{z} u_{\boldsymbol{x}}\right)}_{=2 \varepsilon_{\boldsymbol{x} z}(u)}\|_{T}^{2} \\
& \leq\left\|\left(i d-I_{\boldsymbol{x}, k}^{\mathcal{N}}\right) \varepsilon_{\boldsymbol{x} z}(u)\right\|_{T}^{2}+\left\|I_{\boldsymbol{x}, k}^{\mathcal{N}}\left(i d-I_{z, k}^{\mathcal{N}}\right) \varepsilon_{\boldsymbol{x} z}(u)\right\|_{T}^{2} \\
& \preceq h_{\boldsymbol{x}}^{2 m}\left\|\nabla_{\boldsymbol{x}}^{m} \varepsilon_{\boldsymbol{x} z}(u)\right\|_{\Delta_{T}}^{2}+h_{z}^{2 m}\left\|\nabla_{z}^{m} \varepsilon_{\boldsymbol{x} z}(u)\right\|_{\Delta_{T}}^{2} .
\end{aligned}
$$

Lemma 6. Let $\alpha \in\{\boldsymbol{x}, z\}$, and $F \in \mathcal{F}$ such that its normal lies in direction $\alpha$, i.e. $n=(0,0,1)$ for $\alpha=z$ and $n=\left(n_{x_{1}}, n_{x_{2}}, 0\right)$ for $\alpha=\boldsymbol{x}$. Let $u \in H(\mathrm{curl})$ be in $H^{m+1}\left(\Delta_{T}\right)$ for all $T \in \Delta_{F}$. The tensor product interpolation operator $I_{k}^{\mathcal{N}}$ satisfies the local anisotropic error estimate

$$
\left\|\llbracket u-I_{k}^{\mathcal{N}} u \rrbracket_{n}\right\|_{F}^{2} \preceq \sum_{T \in \Delta_{F}} h_{\alpha}^{2 m+1}\left\|\nabla_{\alpha}^{m} \varepsilon_{\alpha}\left(u_{\alpha}\right)\right\|_{\Delta_{T}}^{2} .
$$

Proof. Let $\beta \in\{\boldsymbol{x}, z\}, \beta \neq \alpha$ be the orthogonal direction to $\alpha$. Due to the choice of $F$, we have $h_{F} \simeq h_{\alpha}$. From the definition of $I_{k}^{\mathcal{N}}$, we see

$$
\left(I_{k}^{\mathcal{N}} u\right)_{\alpha}=I_{\alpha, k}^{\mathcal{N}} I_{\beta, k+1}^{\mathcal{L}} u_{\alpha} .
$$

As the jump in normal direction depends only on the $\alpha$ component, we get by the triangle inequality

$$
\begin{aligned}
\left\|\llbracket u-I_{k}^{\mathcal{N}} u \rrbracket_{n}\right\|_{F} & \leq\left\|\llbracket u_{\alpha}-I_{\alpha, k}^{\mathcal{N}} I_{\beta, k+1}^{\mathcal{L}} u_{\alpha} \rrbracket\right\|_{F} \\
& \leq\left\|\llbracket\left(i d-I_{\beta, k+1}^{\mathcal{L}}\right) u_{\alpha} \rrbracket\right\|_{F}+\left\|\llbracket I_{\beta, k+1}^{\mathcal{L}}\left(i d-I_{\alpha, k}^{\mathcal{N}}\right) u_{\alpha} \rrbracket\right\|_{F} .
\end{aligned}
$$

Due to the linearity of $I_{\beta, k+1}^{\mathcal{L}}$ it commutes with the jump operator for any piecewise smooth function $v$, i.e. $I_{\beta, k+1}^{\mathcal{L}} \llbracket v \rrbracket=\llbracket I_{\beta, k+1}^{\mathcal{L}} v \rrbracket$. As $u$ is supposed to be continuous on $\Delta_{F}$, it is continuous across $F$, and thereby

$$
\left\|\llbracket\left(i d-I_{\beta, k+1}^{\mathcal{L}}\right) u_{\alpha} \rrbracket\right\|_{F}=0
$$


We now concentrate on the second term in the estimate above. Again, using linearity and boundedness in $L^{2}$ of $I_{\beta, k+1}^{\mathcal{L}}$, we obtain

$$
\left\|\llbracket I_{\beta, k+1}^{\mathcal{L}}\left(i d-I_{\alpha, k}^{\mathcal{N}}\right) u_{\alpha} \rrbracket\right\|_{F} \preceq\left\|\llbracket\left(i d-I_{\alpha, k}^{\mathcal{N}}\right) u_{\alpha} \rrbracket\right\|_{F} .
$$

In [31], we provided a Korn-type inequality in the spirit of [23, Theorem 3.1] and the preceding work [12]. It states that, provided a shape regular, simplicial triangulation $\mathcal{T}^{\alpha}$, on a facet $F^{\alpha} \in \mathcal{F}^{\alpha}$

$$
\left\|\llbracket\left(i d-I_{\alpha, 0}^{\mathcal{N}}\right) u_{\alpha} \rrbracket\right\|_{F^{\alpha}}^{2} \preceq h_{\alpha} \sum_{T^{\alpha} \in \Delta_{F^{\alpha}}}\left\|\varepsilon_{\alpha}\left(u_{\alpha}\right)\right\|_{T^{\alpha}}^{2} .
$$

By the hierarchical definition of the Nédélec interpolation operator, we have

$$
I_{\alpha, 0}^{\mathcal{N}} I_{\alpha, k}^{\mathcal{N}} u_{\alpha}=I_{\alpha, 0}^{\mathcal{N}} u_{\alpha} \quad \text { and } \quad \varepsilon\left(I_{\alpha, 0}^{\mathcal{N}} u_{\alpha}\right)=0
$$

When using higher order interpolation operators, one can thus deduce easily

$$
\begin{aligned}
\left\|\llbracket\left(i d-I_{\alpha, k}^{\mathcal{N}}\right) u_{\alpha} \rrbracket\right\|_{F^{\alpha}}^{2} & \preceq h_{\alpha} \sum_{T^{\alpha} \in \Delta_{F^{\alpha}}}\left\|\varepsilon_{\alpha}\left(u_{\alpha}-I_{\alpha, k}^{\mathcal{N}} u_{\alpha}\right)\right\|_{T^{\alpha}}^{2} \\
& \leq h_{\alpha}^{2 m+1} \sum_{T^{\alpha} \in \Delta_{F^{\alpha}}}\left\|\nabla_{\alpha}^{m} \varepsilon_{\alpha}\left(u_{\alpha}\right)\right\|_{\Delta_{T^{\alpha}}}^{2},
\end{aligned}
$$

where we have to use Lemma 4 to obtain estimate (32).

The facet $F$ is the tensor product of a simplicial element $T^{\beta}$ and a facet $F^{\alpha}$. As all functions are supposed to be piecewise smooth, Fubini's theorem is applicable, and integration with respect to $\alpha, \beta$ may be done independently. Using this and the bound (32), we may estimate

$$
\begin{aligned}
\left\|\llbracket\left(i d-I_{\alpha, k}^{\mathcal{N}}\right) u_{\alpha} \rrbracket\right\|_{F}^{2} & =\int_{T^{\beta}} \int_{F^{\alpha}}\left|\llbracket u_{\alpha}-I_{\alpha, k}^{\mathcal{N}} u_{\alpha} \rrbracket\right|^{2} d s_{\alpha} d \beta \\
& \preceq h_{\alpha}^{2 m+1} \sum_{T^{\alpha} \in \Delta_{F}{ }^{\alpha}} \int_{T^{\beta}} \int_{\Delta_{T^{\alpha}}}\left|\varepsilon_{\alpha}\left(u_{\alpha}\right)\right|^{2} d \alpha d \beta \\
& \leq h_{\alpha}^{2 m+1} \sum_{T \in \Delta_{F}}\left\|\varepsilon_{\alpha}\left(u_{\alpha}\right)\right\|_{\Delta_{T}}^{2},
\end{aligned}
$$

which concludes our proof.

Corollary 7. For $m \leq k$ and $u \in\left[H^{m+1}(\Omega)\right]^{d}$, there holds the interpolation error estimate

$$
\left\|u-I_{k}^{\mathcal{N}} u\right\|_{V_{k}}^{2} \preceq h_{\boldsymbol{x}}^{2 m}\left\|\nabla_{\boldsymbol{x}}^{m} \varepsilon(u)\right\|_{\Omega}^{2}+h_{z}^{2 m}\left\|\nabla_{z}^{m} \varepsilon(u)\right\|_{\Omega}^{2} .
$$


Lemma 8. The interpolation operator $I_{k}^{\Sigma}$ defined in (27) satisfies the approximation property

$$
\left\|\sigma-I_{k}^{\Sigma} \sigma\right\|_{T} \preceq h_{\boldsymbol{x}}^{m}\left\|\nabla_{\boldsymbol{x}}^{m} \sigma\right\|_{\Delta_{T}}+h_{z}^{m}\left\|\nabla_{z}^{m} \sigma\right\|_{\Delta_{T}}
$$

for $m \leq k+1$ and $\sigma \in H^{m}\left(\Delta_{T}\right)$ on an element $T \in \mathcal{T}$.

Proof. The result follows directly by bounding the respective subblocks of $\sigma-I_{k}^{\Sigma} \sigma$. We will do the calculations for the $\boldsymbol{x}$-block, the estimate for the other two sub-blocks follows the same line.

$$
\begin{aligned}
\left\|\left(\sigma-I_{k}^{\Sigma} \sigma\right)_{\boldsymbol{x}}\right\|_{T} & =\left\|\sigma_{\boldsymbol{x}}-I_{\boldsymbol{x}, k}^{\Sigma} I_{z, k+1}^{\mathcal{P}} \sigma_{\boldsymbol{x}}\right\|_{T} \\
& \leq\left\|\sigma_{\boldsymbol{x}}-I_{z, k+1}^{\mathcal{P}} \sigma_{\boldsymbol{x}}\right\|_{T}+\left\|I_{z, k+1}^{\mathcal{P}}\left(\sigma_{\boldsymbol{x}}-I_{\boldsymbol{x}, k}^{\Sigma} \sigma_{\boldsymbol{x}}\right)\right\|_{T} .
\end{aligned}
$$

By the approximation properties of the two interpolation operators $I_{\boldsymbol{x}, k}^{\Sigma}$ and $I_{z, k+1}^{\mathcal{P}}$ and the $L^{2}$ continuity of the latter one, we obtain

$$
\left\|\left(\sigma-I_{k}^{\Sigma} \sigma\right)_{\boldsymbol{x}}\right\|_{T} \preceq h_{\boldsymbol{x}}^{m}\left\|\nabla_{\boldsymbol{x}}^{m} \sigma_{\boldsymbol{x}}\right\|_{\Delta_{T}}+h_{z}^{m}\left\|\nabla_{z}^{m} \sigma\right\|_{\Delta_{T}} .
$$

4.3. Anisotropic error estimates. We provide an error estimate for the finite element solution on the tensor product domain. In Theorem 3 we employed standard theory for mixed problems to bound the discretization error by the best-approximation error. Now, estimating this quantity by the interpolation errors from Corollary 7 and Lemma 8, we arrive at the following theorem.

Theorem 9. Let $(u, \sigma)$ be the solution to the continuous problem (8) and $\left(u_{h}, \sigma_{h}\right) \in V_{k} \times \Sigma_{k}$ be the approximation determined by (13). Let $\mathcal{T}$ be a tensor-product mesh for $\Omega$ as defined above. If $u \in H^{m+1}(\Omega)$ and $\sigma \in H^{m}(\Omega)$,

$$
\begin{aligned}
\left\|\sigma-\sigma_{h}\right\|_{\Sigma_{k}}+\left\|u-u_{h}\right\|_{V_{k}} \preceq & h_{\boldsymbol{x}}^{m}\left(\left\|\nabla_{\boldsymbol{x}}^{m} \sigma\right\|_{L^{2}(\Omega)}+\left\|\nabla_{\boldsymbol{x}}^{m} \varepsilon(u)\right\|_{L^{2}(\Omega)}\right)+ \\
& h_{z}^{m}\left(\left\|\nabla_{z}^{m} \sigma\right\|_{L^{2}(\Omega)}+\left\|\nabla_{z}^{m} \varepsilon(u)\right\|_{L^{2}(\Omega)}\right) .
\end{aligned}
$$

We emphasize that the constant hidden in "" is independent of the anisotropic mesh sizes $h_{\boldsymbol{x}}, h_{z}$ and their ratio $\bar{h}_{\boldsymbol{x}} / h_{z}$.

\section{Numerical EXAMPLES}

As a first example, we consider a plate, where the cross section is the unit square, $\Omega_{\boldsymbol{x}}=[0,1] \times[0,1]$. We use a coarse triangular mesh of mesh size $h_{\boldsymbol{x}} \simeq 0.4$. We assume the material to be homogenous and isotropic, and set Young's modulus $E=1$, Poisson ratio $\nu=0.4$. We apply a volume force in vertical direction $f=\left(0,0, d_{z}\right)^{T}$ in $\Omega$. 
We discretize the domain using a coarse tensor-product mesh. The in-plane triangulation $\mathcal{T}^{x}$ consists of 16 elements, in thickness direction we use one to four elements. This implies that the mesh size in thickness direction varies from $h_{z}=d_{z}$ to $h_{z}=0.25 \cdot d_{z}$. We first do all computations for $d_{z}=0.1$, so the aspect ratio of the domain is $1 / 10$. We use adaptive refinement in $\boldsymbol{x}$-direction, but keep the mesh size fixed in z-direction. We use finite element spaces of orders one and two. In Figure 4, we plot the error against the number of degrees of freedom. The different curves correspond to different discretizations with respect to the $z$-direction using one, two and four elements in thickness direction. As expected, a linear or quadratic rate of convergence is achieved in the first refinement steps, indicated by the slope of the error curve almost parallel to the given reference slopes. For the different discretizations with respect to $z$-direction, the error curve saturates at different points. This saturation happens as soon as the total error is dominated by the error due to the mesh size $h_{z}$. In Figure 5 , we used a plate of thickness $d_{z}=0.01$, and one element in thickness direction. Again, we plot the error using adaptive refinement in $\boldsymbol{x}$-direction, and find the almost optimal order of convergence. Moreover, we apply the $p$-version of the finite element method. We use the same coarse mesh as for the $h$-version, but perform one level of geometric refinement towards the sides of the plate. We keep this mesh fixed, and do calculations for increasing polynomial order, namely $k=1, \ldots 4$. We see the exponential convergence one generally expects for high-order methods, although exponential convergence with respect to the polynomial degree was not shown rigorously in the current work. The stability proofs in Section 3 may thus be not optimal.

Our next example is a cylindrical shell of thickness $d_{z}=0.01$. We discretize the shell by prismatic, curved elements, where the order of the element mapping is equal to the order of the underlying finite element spaces. Note that curved elements are not covered by the theory in the previous sections. We used both our mixed finite elements, as well as a standard primal $H^{1}$ conforming discretization. For the standard primal method, we choose elements of polynomial order $k=5$, while for the new mixed method elements of order $k=3$ are used. The standard discretization leads to 49722 coupling degrees of freedom, while for the proposed mixed methods, 42451 coupling degrees of freedom arise. In Figure 6, we plot the absolute value of the stress $|\sigma|$. Clearly, the mixed method leads to better results even though the polynomial order is chosen lower than for the standard method. 


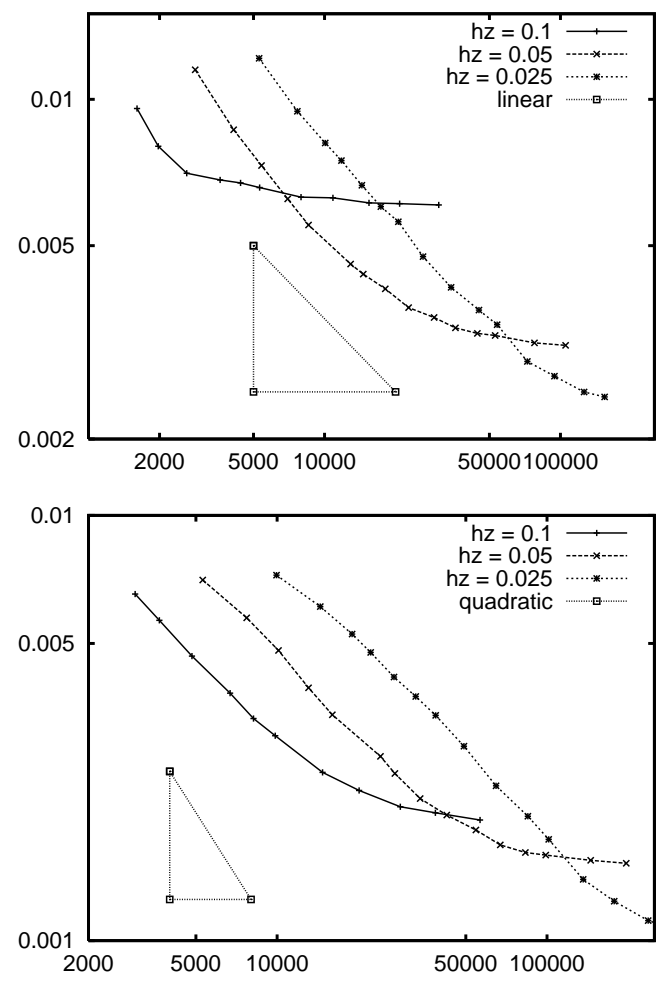

FiguRE 4. Estimated error vs. degrees of freedom for a square plate of thickness $d_{z}=0.1$, with one, two and four elements in transverse direction $\left(h_{z}=0.1,0.05\right.$ and $0.025)$, top order $k=1$, bottom order $k=2$.

\section{ACKNOWLEDGEMENTS}

Both authors acknowledge support from the Austrian Science Foundation FWF within project grant Start Y-192, "hp-FEM: Fast Solvers and Adaptivity"

\section{REFERENCES}

[1] Alessandrini SM, Arnold DN, Falk RS, Madureira AL. Derivation and justification of plate models by variational methods. In Plates and Shells (Quebec 1996), vol. 21 of CRM Proceeding and Lecture Notes, Fortin M (ed). AMS, Providence RI, 1999; 1-20.

[2] Arnold DN, Awanou G, Winther R. Finite elements for symmetric tensors in three dimensions. Math. Comp. 2008; 77(263):1229-1251.

[3] Adams S, Cockburn B. A mixed finite element method for elasticity in three dimensions. J. Sci. Comput. 2005; 25(3):515-521. 


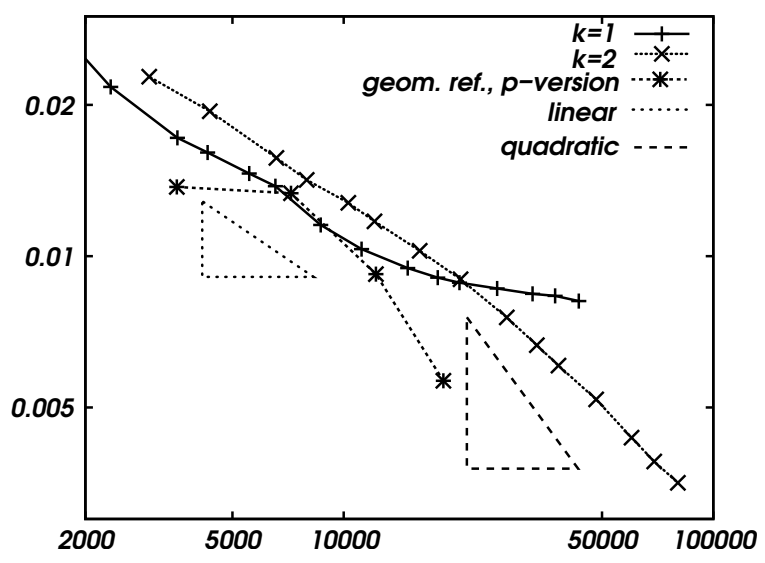

FiguRE 5. Estimated error vs. degrees of freedom for a square plate of thickness $d_{z}=h_{z}=0.01$, order $k=1,2$ with adaptive refinement, and $p$-version FEM (orders $k=1, \ldots 4)$ for prescribed mesh with one level of geometric refinement.
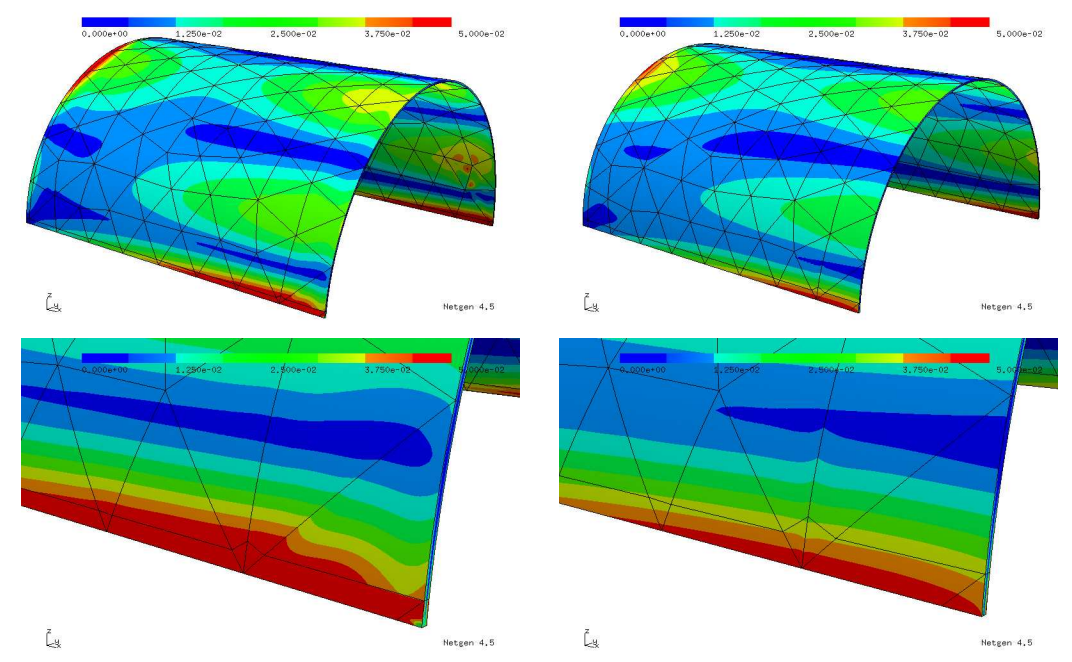

Figure 6. Cylindrical shell, absolute value of the stress $|\sigma|$,range 0 to $0.05 \mathrm{~N} / \mathrm{m}^{2}$, left: standard primal method of order 5, right: new mixed method, order 3

[4] Arnold DN, Falk RS, Winther R. Mixed finite element methods for linear elasticity with weakly imposed symmetry. Math. Comp 2007; 76(260):16991723.

[5] Ainsworth M. A posteriori estimation for fully discrete hierarchical models of elliptic boundary value problems on thin domains. Numer. Math. 1998; 80: 325-362. 
[6] Arnold DN, Winther R. Mixed finite elements for elasticity. Numer. Math 2002; 92:401-419.

[7] Buffa A, Ciarlet P Jr. On traces for functional spaces related to Maxwell's equations Part I: An integration by parts formula in Lipschitz polyhedra. Math Methods Appl. Sci. 2001; 24(1):9-13.

[8] Buffa A, Ciarlet P Jr. On traces for functional spaces related to Maxwell's equations Part II: Hodge decompositions on the boundary of Lipschitz polyhedra and applications. Math Methods Appl. Sci. 2001; 24(1):31-48.

[9] Brezzi F, Fortin M. Mixed and Hybrid Finite Element Methods. SpringerVerlag: New York, 1991.

[10] Babuška I, Li L. Hierarchic modeling of plates. Computers and Structures 1991; 40:419-430.

[11] Braess D. Finite Elemente: Theorie, schnelle Löser und Anwendungen in der Elastizitätstheorie. Springer Verlag: Berlin, 1992.

[12] Brenner S. Korn's inequalities for piecewise $H^{1}$ vector fields. Mathematics of Computation 2004; 73:1067-1087.

[13] Brenner SC, Scott LR. The Mathematical Theory of Finite Element Methods. Springer-Verlag: New York, 2002.

[14] Clément P. Approximation by finite element functions using local regularization. R.A.I.R.O. Anal. Numer. 1975; 9:77-84.

[15] Düster A, Bröker H, Rank E. The p-version of the finite element method for three-dimensional curved thin walled structures. Int. J. Num. Meth. Eng. 2001; 52:673-703.

[16] Demkowicz L, Kurtz J, Pardo D, Paszyński M, Rachowicz W, Zdunek A. Computing with hp-adaptive finite elements. Vol. 2. Chapman \& Hall/CRC Applied Mathematics and Nonlinear Science Series, 2008.

[17] Duvaut G, Lions JL. Inequalities in Mathematics and Physics. Springer-Verlag: Berlin Heidelberg New York, 1976.

[18] Dupont T, Scott R. Polynomial approximation of functions in Sobolev spaces. Math. Comp. 1980; 34(150):441-463.

[19] Koiter WT. A consistent first approximation in the general theory of thin elastic shells. In Proc. Sympos. Thin Elastic Shells (Delft, 1959). North-Holland: Amsterdam, 1960; 12-33.

[20] Dauge M, Faou E, Yosibash Z. Plates and shells: Asymptotic expansions and hierarchical models. In Encyclopedia of Computational Mechanics. Volume I. Stein E, de Borst R, Hughes TJR (eds). 2004; 199-236.

[21] Mindlin RD. Influence of rotatory inertia and shear flexural motions of isotropic elastic plates. J. Appl. Mech. 1951; 18:31-38.

[22] Monk P. Finite element methods for Maxwell's equations. Claredon Press: Oxford, 2003.

[23] Mardal KA, Winther R. An observation on Korn's inequality for nonconforming finite element methods. Math.Comp. 2006; 75(253):1-6.

[24] Nédélec JC. Mixed finite elements in $\mathbb{R}^{3}$. Numer. Math. 1980; 35:315-341.

[25] Nédélec JC. A new family of mixed finite elements in $\mathbb{R}^{3}$. Numer. Math 1986; 50:57-81.

[26] Nitsche JA. On Korn's second inequality. RAIRO Anal. Numér. 1981; 15(3):237-248. 
[27] Reissner E. The effect of transverse shear deformation on the bending of elastic plate models. J. Appl. Mech. 1945; 12:69-76.

[28] Reissner E. On a variational theorem in elasticity. J. Math. Physics 1950; 29:90-95.

[29] Schwab C. A-posteriori modeling error estimation for hierarchic plate models. Numer. Math. 1996; 74:221-259.

[30] Schöberl J. Commuting quasi-interpolation operators for mixed finite elements. Report ISC-01-10-MATH, Texas A\&M University; 2001.

[31] Sinwel A. A New Family of Mixed Finite Elements for Elasticity. PhD thesis, Institut für Numerische Mathematik, Johannes Kepler University Linz, Austria, 2009.

[32] Stein E, Ohnimus S. Coupled model- and solution-adaptivity in the finiteelement method. Comput. Methods Appl. Mech. Engrg., Symposium on Advances in Computational Mechanics, Vol. 2 (Austin, TX, 1997) 1997; 150(14):327-350.

[33] Schöberl J, Sinwel A. Tangential-Displacement and Normal-Normal-Stress Continuous Mixed Finite Elements for Elasticity. submitted.

[34] Scott LR, Zhang S. Finite element interpolation of nonsmooth functions satisfying boundary conditions. Math. Comp 1990; 54(190):483-493.

[35] Schöberl J, Zaglmayr S. High order Nédélec elements with local complete sequence properties. COMPEL 2005; 24(2):374-384.

[36] Timoshenko SP, Woinowsky-Krieger S. Theory of plates and shells (2nd ed). Engineering Societies Monographs, New York: McGraw-Hill, 1959.

[37] Vogelius M, Babuška I. On a dimensional reduction method. I. The optimal selection of basis functions. Math. Comp. 1981; 37(155):31-46.

[38] Zaglmayr S. High Order Finite Element Methods for Electromagnetic Field Computation. PhD thesis, Institut für Numerische Mathematik, Johannes Kepler University Linz, Austria, 2006.

1 Institute of Technical Mechanics, Johannes Kepler University Linz, Altenbergerstr. 69, 4040 Linz, Austria,, ${ }^{2}$ Institute for Analysis and Scientific Computing, Vienna University of Technology, Wiedner Hauptstrasse 8-10, 1040 Wien, Austria 